\title{
Review
}

\section{Pioneering Role of Marine Macroalgae in Cosmeceuticals}

\author{
Haresh S. Kalasariya ${ }^{1, *(D)}$, Leonel Pereira ${ }^{2, *(D)}$ and Nikunj B. Patel ${ }^{1}$ \\ 1 Microbiology Department, Sankalchand Patel University, Visnagar 384315, Gujarat, India; \\ nbpatel.fsh@spu.ac.in \\ 2 MARE-Marine and Environmental Sciences Centre, Department of Life Sciences, University of Coimbra, \\ Calçada Martim de Freitas, 3000-456 Coimbra, Portugal \\ * Correspondence: hskalasariya.fsh@spu.ac.in (H.S.K.); leonel.pereira@uc.pt (L.P.)
}

\section{check for}

updates

Citation: Kalasariya, H.S.; Pereira, L.; Patel, N.B. Pioneering Role of Marine Macroalgae in Cosmeceuticals. Phycology 2022, 2, 172-203. https:// doi.org/10.3390/phycology2010010

Academic Editor: Peer Schenk

Received: 29 December 2021

Accepted: 16 February 2022

Published: 1 March 2022

Publisher's Note: MDPI stays neutral with regard to jurisdictional claims in published maps and institutional affiliations.

Copyright: (C) 2022 by the authors. Licensee MDPI, Basel, Switzerland. This article is an open access article distributed under the terms and conditions of the Creative Commons Attribution (CC BY) license (https:// creativecommons.org/licenses/by/ $4.0 /)$.

\begin{abstract}
Cosmetics are broadly used by people to protect the skin from external environmental stresses and for beauty purposes globally. A recent trend towards cosmetics with natural formulations has emerged. The cosmetic industry uses the term 'cosmeceutical' to refer to a cosmetic formula that has drug-like applicative advantages. Recently, macroalgae have received increased attention as natural ingredients for cosmeceutical applications. Many marine algae are rich in biologically active components that have been reported to exhibit strong benefits to the skin, mainly for photoprotection, skin whitening, moisturization, anti-aging, anti-wrinkle, antioxidants, and antimicrobial uses. The present review provides a detailed study of the literature on the cosmetic potentials of marine algaederived polysaccharides, peptides and amino acids, pigments, phenolic components, and fatty acids. We provide an overview of different types of macroalgae with their biologically active constituents and potential cosmetic benefits. In addition, the bioactive molecules of cosmetic products containing marine macroalgae as well as their mechanisms of action are briefly discussed.
\end{abstract}

Keywords: seaweeds; bioactivity; cosmeceuticals; skin care; anti-inflammatory; photoprotection

\section{Introduction}

In cosmeceuticals, cosmetic products are a topical combination of cosmetic and pharmaceutical with bioactive molecules to have medicinal or drug-like applications to improve health and texture of skin [1,2]. Due to modernization and skin care attention, cosmetic companies are enlarging gradually each year worldwide. To fulfill the requirements of customers, these cosmetic companies are moving towards unbeatable exploitation of synthetic cosmetics and constituents. Due to the ineffectiveness of synthetic components, it may cumulate in skin and produce toxic effects and may cause harm to healthy skin structure. Hydroxybenzoic acid esters (parabens) reported its adverse effect to the skin as well as increase incidence of malignant melanoma and breast cancer since it is widely used in cosmetic formulations [3]. Another substance is phthalate, which is highly found in different cosmetic formulations that can cause DNA mutations and damage, as found in human male gamete [4,5]. Some of these synthetic chemical compounds can cause detrimental effects in animals such as reduction of sperm counts, changed pregnancy outcomes, congenital disabilities of male genitalia, etc. [6]. As a result, users have changed their liking and selected natural cosmetic products for usage [7]. Hence, the enlarging market for skincare formulations and constant look for an alternative natural constituents led to the production of a different types of cosmeceutical skin products [8]. Cosmetics has had a commanding role in society, mainly for traditional and conventional purposes, since immemorial times. In ancient times, people were aware of natural compounds extracted from milk, flowers, fruits, seed, vegetables, herbs, marine algae, and minerals from clay, ash, etc. [5]. In different parts of the world, there is still a practice of utilizing marine macroalgae as an alternative natural sustainable remedy by making it into a promising natural raw material in skin cosmetics [9]. 
Marine macroalgae (seaweeds) are macroscopic, multicellular, eukaryotic organisms that can perform photosynthesis due to presence of Chlorophyll and some other photosynthetic pigments. They are widely distributed along the coastal line (the intertidal and sub-tidal regions) and in brackish water [10]. Based on pigment composition, they can be classified into three types. Brown alga belongs to Ochrophyta phylum (Phaeophyceae class), red alga belongs to Rhodophyta phylum, and green alga belongs to Chlorophyta phylum. Among these three types, brown algae belong to the Chromista kingdom, whereas green and red algae belong to the Plantae kingdom $[10,11]$. Seaweeds have a more highly diversified bioactive constituents than terrestrial organisms [12]. These bioactive compounds have a wide range of biological activities which can be used in product preparation as an ingredient $[13,14]$. The applications of macroalgae in cosmeceutical formulations depends on their constituents (such as polysaccharides, carbohydrate derivatives, proteins, peptides, amino acids, phenolic compounds, vitamins, minerals, fatty acids, pigments, etc.) $[15,16]$. Many previous findings have reported the role of seaweed based bioactive compounds which offer antitumor, antiallergic, antimicrobial, antioxidant, antiinflammation, antilipidemic activity, antiwrinkle, anti-aging, moisturizing, and photoprotection activities [5,15,17-19]. The present study aims to reveal the role of marine macroalgae-derived polysaccharides, peptides, and amino acids, pigments, phenolic compounds as well as lipid and fatty acids in skin cosmetic benefits.

\section{Seaweed Derived Metabolites in Cosmetics}

Due to presence of the above-mentioned bioactive constituents and their potential biological activities, they can be broadly use in cosmeceutical products. For the preparation of cosmeceutical products, macroalgae-derived compounds have been noted as being of significant importance [20]. Polysaccharides have a great role in cosmetics including in moisturizers, emulsifiers, wound healing agents, and thickening agents [21]. Fernando et al. [22] have reported anti-inflammation activity of Fucoidan from Chnoospora minima (Phaeophyceae) by inhibition of Lipopolysaccharides induced nitric oxide production, inducible nitric oxide productions, Cyclooxygenase-2, and Prostaglandin E2 levels in an experimental study by targeting RAW macrophages. Likewise, Ariede et al. [23], Wang et al. [24], and Teas and Irhimeh, [25] reported beneficial activities of Fucus vesiculosus (Figure 1a) (Phaeophyceae) derived polysaccharides such as anti-aging, anti-melanogenic, anti-cancer, and antioxidant activity by stimulating collagen production, tyrosinase inhibition, decreasing melanoma growth and by preventing oxidation formation, respectively. In addition, the anti-inflammation activity of sulphated polysaccharide from Padina tetrastromatica (Phaeophyceae) by COX-2 and iNOS inhibitions in an experimental model of Paw edema in rats [26]. Moreover, Khan et al. [27] reported the anti-inflammation activity of polyunsaturated fatty acids derived from Undaria pinnatifida (Figure 1b) (Phaeophyceae) on mouse ear edema and erythema. In vitro, the antioxidant activity of methanolic extracts from Osmundaria obtusilo and Palisada flagellifera (Rhodophyta) was studied by DPPH, ABTS, metal chelating, Folin ciocalteau, and beta-carotene bleaching assays [28,29]. Phenolic compound Sargachromanol E revealed antiaging activities from Sargassum horneri (Phaeophyceae) by inhibition of matric metalloprotein expression on UVA irradiated dermal fibroblast [30]. Likewise, the Anti-melanogenic activity of Sargachromanol E from Sargassum serratifolium (Phaeophyceae) was studied by downregulation of microphthalmiaassociated transcription factors on B16F10 melanoma cells [31]. Along with this, Yoon et al. [32] showed inhibition of tyrosinase and melanin and expressed anti-melanogenic activity of the brown macroalga Petalonia binghamiae's ethanolic extract. Besides, mycosporine amino acids (MAAs) from Porphyra sp. (Rhodophyta) reported collagenase inhibition as well as control expression of MMP (on human fibroblast cell lines) in vitro [33]. Additionally, antioxidant activity revealed by Neopyropia yezoensis (formerly Porphyra yezoensis) (Rhodophyta) derived Mycosporine amino acids by ROS scavenging potential and MMP expression on human skin fibroblast [34]. The antiaging activity was found by collagenase inhibition of Palmaria palmata (Figure 1g) (Rhodophyta) derived MAAs [35]. Some species 
of macroalgae Laurencia pacifica, Laurencia rigida, Wilsonosiphonia howei (formerly Polysiphonia howei), Rhodomela confervoides, Schizymenia dubyi (Figure 1c) (Rhodophyta), etc. were found to be a good source of phenolic compounds $[11,31,36]$.
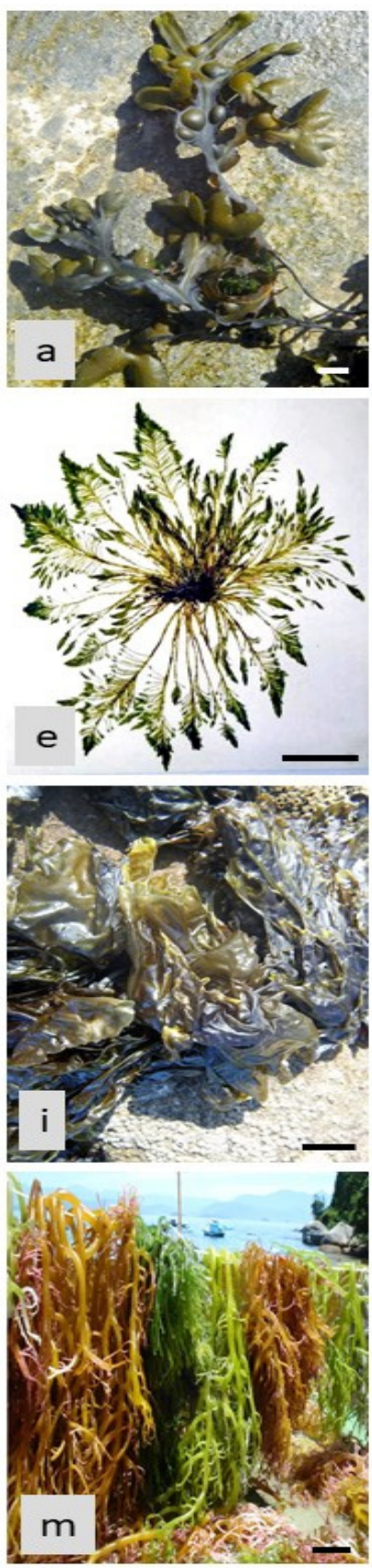
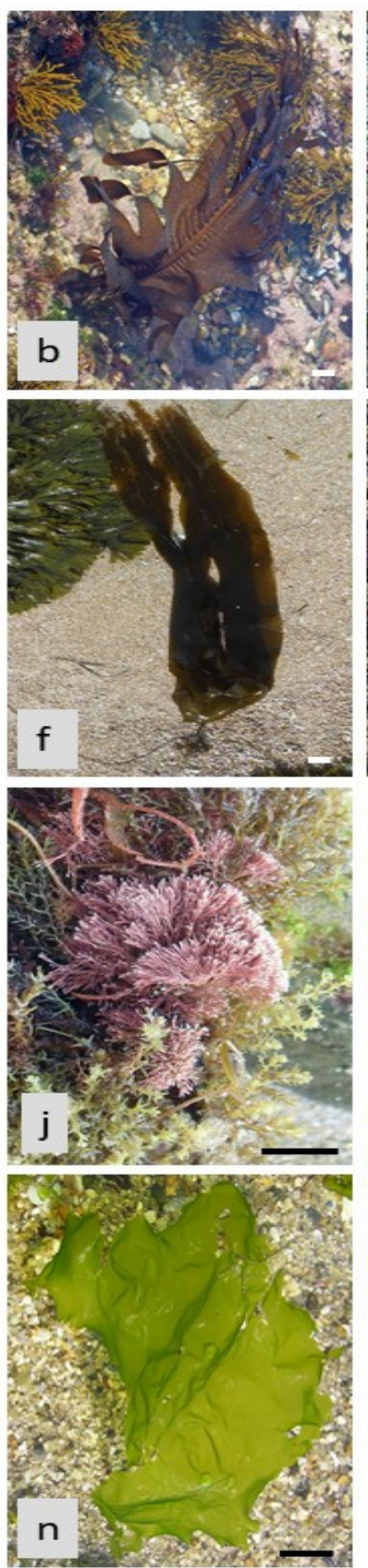
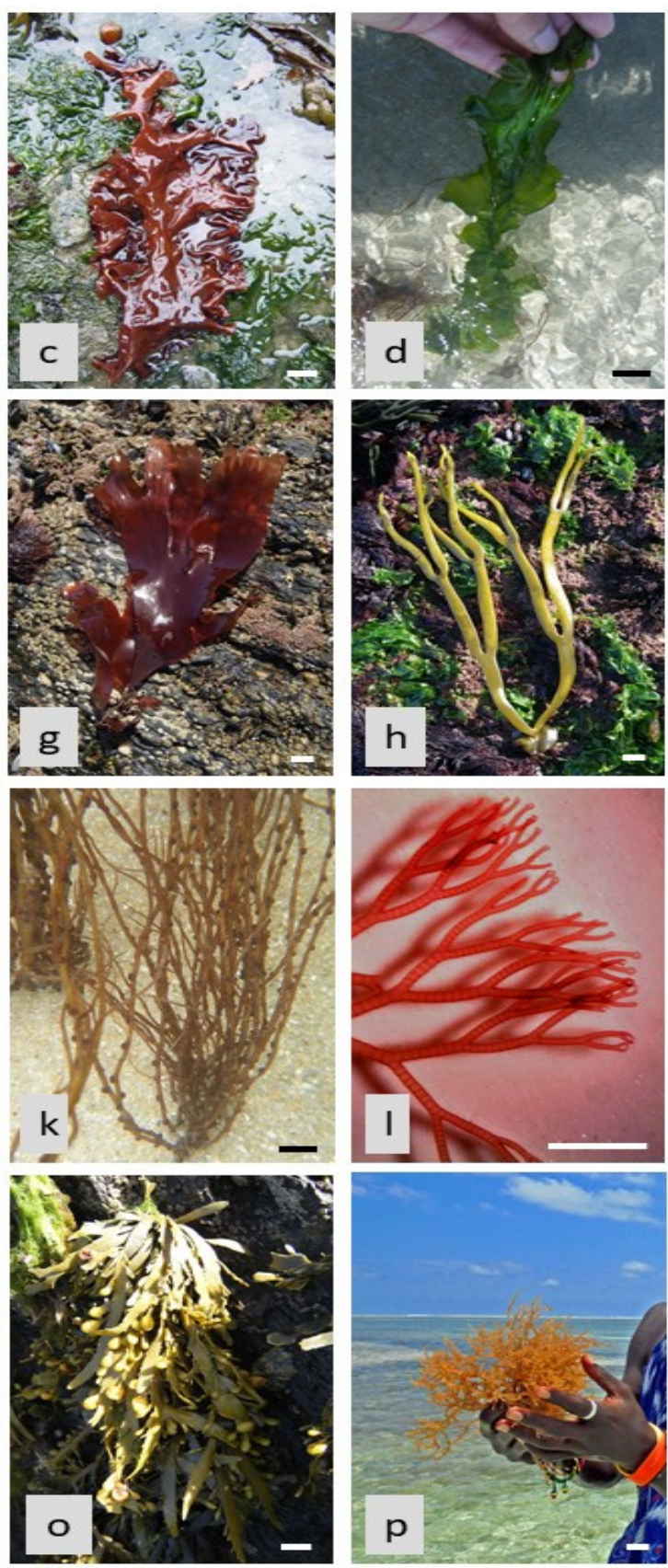

Figure 1. Seaweed species images: (a)—Fucus vesiculosus (P); (b)—Undaria pinnatifida (P); (c)-Schizymenia dubyi (R); (d) -Ulva linza (C); (e)—Bryopsis plumosa (C); (f) - Laminaria digitata (P); (g)—Palmaria palmata (R); (h)—Himanthalia elongata (P); (i)—Porphyra umbilicalis $(\mathrm{R})$; (j)—Jania rubens (R); (k)—Gracilaria gracilis (R); (l)—Ceramium virgatum (R); (m)—Kappaphycus alvarezii (R); (n) —Ulva lactuca (C); (o)—Ascophyllum nodosum (P); (p) —Eucheuma denticulatum (R); C-Chlorophyta; R-Rhodophyta; P-Phaeophyceae; Scale $=1 \mathrm{~cm}$.

\section{Polysaccharides}

Marine macroalgae derived polysaccharides are well known for their biological benefits. Many previous research studies have suggested the presence of polysaccharides (ulvan, fucoidan, alginate, laminarin, carrageenan, sulphated polysaccharides, agar, and 
agarose) in macroalgae and noted their cosmeceutical benefits. Other examples of macroalgae derived polysaccharides and their cosmetic benefits are presented in Table 1. Wang et al. [37] suggested important bioactivities of fucoidan (including antioxidant, antimicrobial, anti-inflammatory, anticancer, antihyperlipidemic, and other bioactivities). Some studies suggested the utilization of polysaccharides as bioactive constituent in skin care formulations, played a major role in cosmetics such as moisturizers, emulsifiers, wound healing, and as a thickener [38]. The chemical structures of marine algae derived polysaccharides are illustrated in Figure 2.

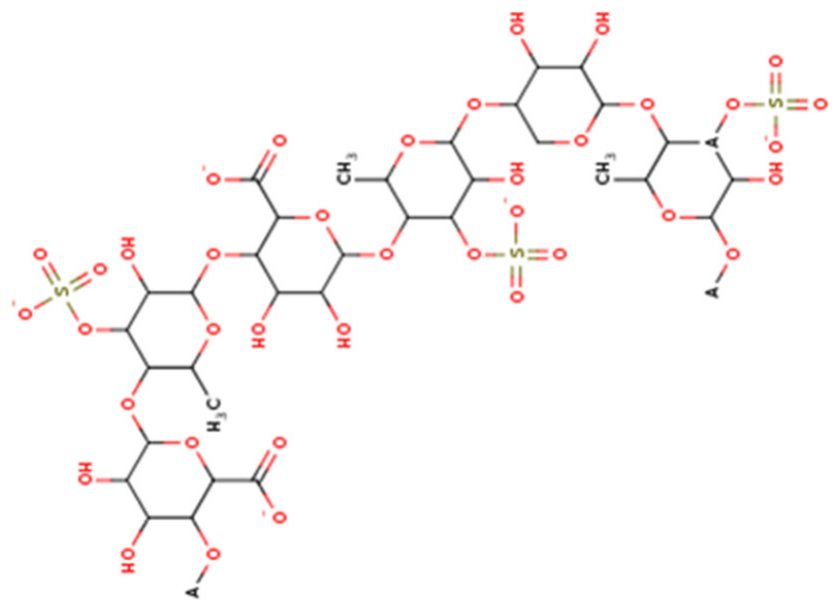

(a)

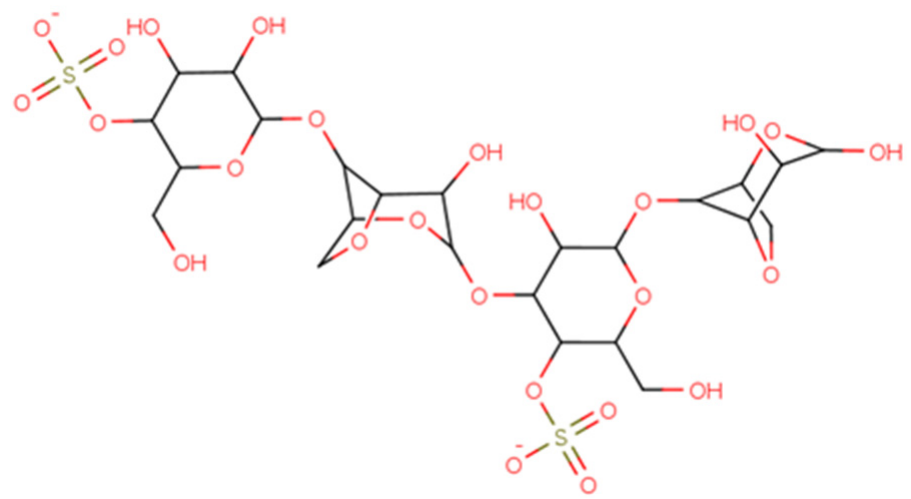

(c)

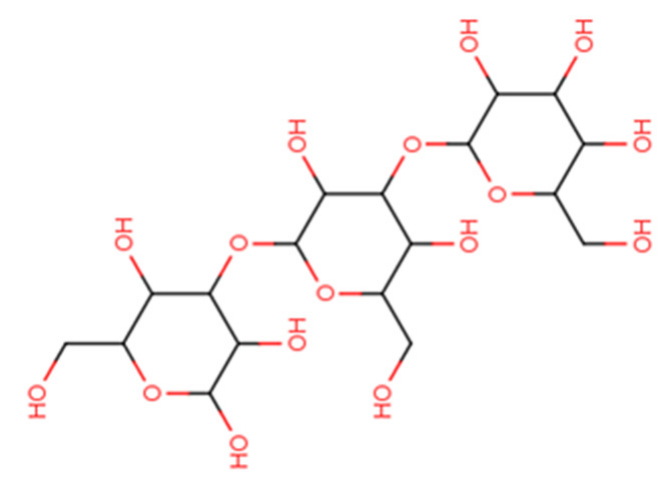

(e)<smiles>COC1C(O)C(CO)OC(OC2C(O)C3OCC2C3O)C1O</smiles>

(b)<smiles>COC1OC(CO)C(O)C(OC2OC(COS(=O)(=O)[O-])C(OC3OC(CO)C(O)C(OC4OC(CO)C(OC)C(O)C4O)C3O)C(O)C2O)C1OS(=O)(=O)[O-]</smiles>

(d)<smiles>COC1C(C)OC(C)C(OS(=O)(=O)O)C1O</smiles>

$(\mathbf{f})$

Figure 2. Chemical structures of marine macroalgae derived polysaccharides. (a) Ulvan, (b) agar, (c) kappa-carrageenan, (d) porphyran, (e) laminaran, and (f) fucoidan. 
Table 1. Application of macroalgae derived polysaccharides in skin cosmetics.

\begin{tabular}{|c|c|c|c|c|}
\hline No. & Name of Macroalgae & Polysaccharides & Cosmetic Benefits & References \\
\hline \multirow[t]{2}{*}{1} & $\begin{array}{c}\text { Ulva lactuca } \\
\text { (Figure 1n) (C) }\end{array}$ & SP (Ulvan) & $\begin{array}{l}\text { Antioxidant, Moisturizer, } \\
\text { Photoprotective }\end{array}$ & [39] \\
\hline & Neopyropia yezoensis (R) & Porphyran & Antiinflammation & {$[40,41]$} \\
\hline 2 & $\begin{array}{c}\text { Porphyridium sp.* }{ }^{*}(\mathrm{R}) \\
\text { Costaria costata }(\mathrm{P}), \text { Ulva lactuca } \\
\text { (Figure } 1 \mathrm{n})(\mathrm{C})\end{array}$ & $\begin{array}{c}\text { Sulphated } \\
\text { polysaccharides }\end{array}$ & $\begin{array}{c}\text { Antioxidant, } \\
\text { Anti-inflammatory, } \\
\text { Antiaging }\end{array}$ & [42] \\
\hline 3 & Fucus vesiculosus (Figure 1a) & Fucoidans & Antiaging, Antiwrinkle & [43] \\
\hline 4 & $\begin{array}{c}\text { Ascophyllum nododum } \\
\text { (Figure 1o), } \\
\text { Chnoospora minima, } \\
\text { Sargassum fusiforme, } \\
\text { Saccharina japonica, Sargassum } \\
\text { polycystum, } \\
\text { S. vachellianum, } \\
\text { S. hemiphyllum }(\mathrm{P})\end{array}$ & Fucoidans & $\begin{array}{c}\text { Photoprotection, Anti } \\
\text { photoaging } \\
\text { Anti-inflammatory, } \\
\text { Anti-elastase, Anti-collagenase, } \\
\text { Skin whitening }\end{array}$ & [44-47] \\
\hline 5 & $\begin{array}{l}\text { Fucus vesiculosus } \\
\text { (Figure } 1 \mathrm{a})(\mathrm{P})\end{array}$ & Fucoidan & $\begin{array}{l}\text { Anticoagulant Antioxidant, } \\
\text { Enhancer of Skin fibroblast } \\
\text { formation }\end{array}$ & [48] \\
\hline 6 & Neoporphyra haitanensis (R) & Porphyran & Antioxidant & {$[49,50]$} \\
\hline 7 & Saccharina longicruris $(\mathrm{P})$ & Laminaran & $\begin{array}{c}\text { Anti-inflammation, Antioxidant, } \\
\text { Reconstruction of dermis }\end{array}$ & {$[51,52]$} \\
\hline 8 & Saccharina longicruris $(\mathrm{P})$ & Galactofucans & $\begin{array}{l}\text { Enhance fibroblast formation, } \\
\text { Increase synthesis of matrix } \\
\text { metalloproteinase (MMP) } \\
\text { complex and collagen-1 }\end{array}$ & [53] \\
\hline 9 & $\begin{array}{l}\text { Eucheuma denticulatum } \\
\quad \text { (Figure } 1 \mathrm{p})(\mathrm{R})\end{array}$ & Carrageenan & Antioxidant, photoprotection & [54] \\
\hline 10 & Gelidium sp. (R) & Agar & Thickener & [55] \\
\hline 11 & $\begin{array}{c}\text { Ascophyllum sp., } \\
\text { Fucus sp., } \\
\text { Sargassum sp., Undaria sp. (P) }\end{array}$ & Laminaran & Anticellulite & [56] \\
\hline 12 & Saccharina cichorioides $(\mathrm{P})$ & Fucoidan & Anti-atopic dermatitis & [57] \\
\hline 13 & $\begin{array}{l}\text { Corallina officinalis } \\
\text { (Figure } 4 \mathrm{a})(\mathrm{R})\end{array}$ & $\begin{array}{c}\text { Sulphated } \\
\text { polysaccharides }\end{array}$ & Antioxidant & [58] \\
\hline 14 & Ulva australis $(\mathrm{C})$ & Ulvan & Antiaging & {$[59,60]$} \\
\hline 15 & Acanthophora muscoides (R) & $\begin{array}{l}\text { Sulphated } \\
\text { polysaccharides- } \\
\text { Carrageenan }\end{array}$ & $\begin{array}{l}\text { Anticoagulant, Antinociceptive, } \\
\text { antiinflammation, Gel agents }\end{array}$ & [61-63] \\
\hline 17 & Chondrus crispus (R) & Carrageenan & $\begin{array}{c}\text { Gel and Thickening agent, Skin } \\
\text { moisturizer }\end{array}$ & {$[64]$} \\
\hline 18 & $\begin{array}{l}\text { Ulva rigida (Figure } 4 \mathrm{~m}) \text {, } \\
\text { U. pseudorotundata }(\mathrm{C})\end{array}$ & $\begin{array}{c}\text { Sulphated } \\
\text { polysaccharides }\end{array}$ & $\begin{array}{c}\text { Antioxidant, Chelators, Gel } \\
\text { agents, Moisturizer }\end{array}$ & {$[65]$} \\
\hline 19 & $\begin{array}{l}\text { Ascophyllum nodosum } \\
\quad \text { (Figure } 10)(\mathrm{P})\end{array}$ & Fucoidan & $\begin{array}{c}\text { Anti-inflammation, Antiviral, } \\
\text { Antiaging, Anti elastase, } \\
\text { Photoprotective, Tyrosinase } \\
\text { inhibition, Anticellulite }\end{array}$ & [66] \\
\hline 20 & Gracilaria sp. (R) & Agar & Thickener & [67] \\
\hline 21 & Padina boergesenii (P) & $\begin{array}{c}\text { Sulphated } \\
\text { polysaccharides }\end{array}$ & Formation of collagen & {$[68]$} \\
\hline
\end{tabular}


Table 1. Cont.

\begin{tabular}{|c|c|c|c|c|}
\hline No. & Name of Macroalgae & Polysaccharides & Cosmetic Benefits & References \\
\hline 22 & $\begin{array}{l}\text { Macrocystis sp., Lessonia sp., } \\
\text { Laminaria sp. (P) }\end{array}$ & Alginate & $\begin{array}{l}\text { Gelling and Stabilizing agent, } \\
\text { Moisturizer, Chelator }\end{array}$ & {$[69,70]$} \\
\hline 24 & Kjellmaniella crassifolia & Fucoidan & Antiaging, Antiwrinkle & [71] \\
\hline 25 & Brown algae $(\mathrm{P})$ & Alginate & $\begin{array}{l}\text { Thickening agent } \\
\text { Gelling agent }\end{array}$ & [72] \\
\hline 27 & $\begin{array}{c}\text { Sargassum } \\
\text { vachellianum }(\mathrm{P})\end{array}$ & Polysaccharides & Skin moisturizer and protectors & [73] \\
\hline 28 & $\begin{array}{c}\text { Fucus vesiculosus (Figure 1a), } \\
\text { Laminaria digitata (Figure 1f), } \\
\text { Undaria pinnatifida (Figure 1b) (P) }\end{array}$ & Fucoidan & $\begin{array}{l}\text { Antioxidant, Antiaging, } \\
\text { Anticoagulant, Increase skin } \\
\text { fibroblast formulation }\end{array}$ & {$[74,75]$} \\
\hline 29 & $\begin{array}{l}\text { Ascophyllum nodosum } \\
\quad \text { (Figure 1o) }(\mathrm{P})\end{array}$ & Fucoidan & $\begin{array}{c}\text { Anti-elastase, gelatinase A } \\
\text { inhibition, Inhibition of } \\
\text { interleukin-1 beta in fibroblast } \\
\text { cells }\end{array}$ & [76] \\
\hline 30 & Ecklonia cava $(\mathrm{P})$ & Phlorotannins & Photoprotectors against UV-B & {$[77,78]$} \\
\hline 31 & $\begin{array}{l}\text { Neoporphyra haitanensis, } \\
\text { Gracilaria chouae, } \\
\text { G. blodgetti (R) }\end{array}$ & Agar & $\begin{array}{c}\text { Antioxidant, } \\
\text { Thickeners } \\
\text { Antitumor, } \\
\text { Radiation protector, } \\
\text { Antiaging }\end{array}$ & {$[79,80]$} \\
\hline 32 & Turbinaria conoides $(\mathrm{P})$ & $\begin{array}{c}\text { Laminarin, Alginate, } \\
\text { Fucoidan }\end{array}$ & Antioxidant & {$[81]$} \\
\hline
\end{tabular}

SP, Sulphated Polysaccharides; C, Chlorophyta; R, Rhodophyta; P, Phaeophyceae; * Microalgae.

Many previous studies demonstrated that fucoidan obtained from brown algae, Fucus sp., Sargassum sp., as well as brown alga, Laminaria sp. (Phaeophyceae) can be utilized as an inhibitor of tyrosinase [82]. Brown algae such as Chnoospora minima and Sargassum polycystum derived fucoidan can inhibit tyrosinase, collagenase, and elastase [83]. Park and Choi, [84] also demonstrated the improved inhibition of low molecular weight fucoidan in a melanoma cell.

Polysaccharides report a key role in collagenase and elastase inhibition. Sulphated polysaccharides obtained from brown alga, S. fusiforme (formerly Hizikia fusiformis) (Phaeophyceae) potentially inhibited collagenase and elastase by regulating different pathways in HDF cells radiated by UVB [85]. In a dose-dependent manner, Chnoospora minima and Sargassum polycystum derived fucoidan showed elastase and collagenase inhibition activities [40]. Furthermore, Moon et al. [86,87] studied the inhibitory activity of MMP1 promoter and increase the expression of Type-1 procollagen synthesis of Fucoidan.

Polysaccharides have the greatest amount of water retention time and can act as humectant and moisturizer in cosmetic products. Many research studies suggested greater hydration capacity and moisturizing effects of polysaccharides from Saccharina japonica (formerly Laminaria japonica) (Phaeophyceae) than hyaluronic acid [88]. Isolation of sulphated polysaccharide was carried out from the green macroalgae Ulva linza (formerly Ulva fasciata) which revealed higher moisture absorption and retention for $96 \mathrm{~h}$ in comparison to glycerol [89]. Saccharina japonica, Neoporphyra haitanensis (formerly Porphyra haitanensis) (Rhodophyta), Codium fragile, Ulva linza (Figure 1d) (formerly Enteromorpha linza), and Bryopsis plumosa (Figure 1e) (Chlorophyta) derived polysaccharides showed good amount of moisture absorption and retention [90]. They also reported the role of sulphate content and molecular weight in the moisture-holding capacity.

Oligosaccharide zinc complex derived from Laminaria digitata (Figure 1f) (Phaeophyceae) reported anti-acne activity (i.e., reduction of acne signs) by reducing sebum pro- 
duction [91]. Sebaaly et al. [92] reported bactericidal activity of Corallina sp. (Rhodophyta) derived sulphated galactan against Enterococcus faecalis and Streptococcus epidermidis.

\section{Amino Acids}

Protein is considered a macromolecule and polymer of amino acids. Pereira [76] reported the role of amino acids as a natural moisturizing factor that prevents water loss in the skin. Marine macroalgae are a satisfactory resource of various amino acids, such as glycine, alanine, valine, leucine, proline, arginine, serine, histidine, tyrosine, and some other mycosporine amino acids (MAAs). Marine macroalgae derived peptides and amino acids and its skin cosmetic benefits are illustrated in Table 2. In cosmeceutical products, amino acids usually function as a hydrating agent as a natural moisturizing factor in human skin [93].

Stengel et al. [94] reported that macroalgae are a quality source of essential amino acids, such as histidine, tyrosine, tryptophan, and non-essential amino acids such as alanine, serine, and proline. Antioxidant activity was reported by Ulva australis-derived amino acids, histidine, and taurine [95]. On the other hand, red macroalgae Palmaria palmata (Figure 1g) (Dulse) and brown alga Himanthalia elongata (Figure 1h) are plentiful in glutamic acid, alanine, and serine [96]. The chemical structures of marine algae derived amino acids are illustrated in Figure 3.<smiles>CC(N)C(=O)O</smiles>

(a)<smiles>NC(Cc1cnc[nH]1)C(=O)O</smiles>

(d)<smiles>NC(Cc1c[nH]c2ccccc12)C(=O)O</smiles>

(f)

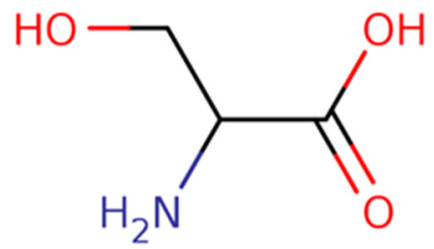

(b)

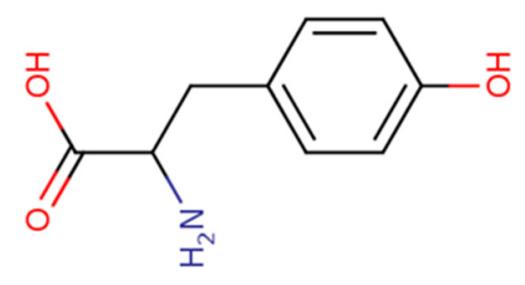

(e)<smiles>NC(CCC(=O)O)C(=O)O</smiles>

(g)<smiles>COC1=C(NCC(=O)O)CC(O)(CO)CC1=O</smiles>

(h)

Figure 3. Chemical structures of amino acids derived from marine macroalgae. (a) Alanine, (b) serine, (c) proline, (d) histidine, (e) tyrosine, (f) tryptophan, (g) glutamic acid, and (h) mycosporine glycine. 
Table 2. Applications of macroalgae derived peptides and amino acids in skin cosmetics.

\begin{tabular}{|c|c|c|c|c|}
\hline No. & Name of Macroalgae & Compounds & Cosmetic Benefits & References \\
\hline 1 & Scytosiphon lomentaria $(\mathrm{P})$ & Amino acids & $\begin{array}{l}\text { Antioxidant, Radical scavengers, } \\
\text { Chelators }\end{array}$ & [97-99] \\
\hline 2 & Gracilaria vermiculophylla (R) & $\begin{array}{c}\text { Porphyra-334, } \\
\text { Palythine, Asterina-330, } \\
\text { Shinorine }\end{array}$ & Antioxidant, UV protector & [100] \\
\hline 3 & $\begin{array}{l}\text { Ulva lactuca (Figure } 1 \mathrm{n})(\mathrm{C}), \\
\text { Asparagopsis armata } \\
\text { (Figure } 4 \mathrm{c})(\mathrm{R})\end{array}$ & MAAs, Amino acids & $\begin{array}{l}\text { Antiaging, Anti wrinkles, } \\
\text { Improves collagen formation }\end{array}$ & [101] \\
\hline 4 & $\begin{array}{l}\text { Pelvetia canaliculata } \\
\quad(\text { Figure } 4 \mathrm{~d})(\mathrm{P})\end{array}$ & Amino acids & $\begin{array}{l}\text { Antioxidant, Collagen formation, } \\
\text { Proteoglycan's synthesis }\end{array}$ & [102] \\
\hline 5 & $\begin{array}{c}\text { Gracilaria chilensis, Pyropia plicata, } \\
\text { Champia novae-zelandiae }(\mathrm{R})\end{array}$ & MAAs & Anti UV, Antioxidant & [103] \\
\hline 6 & $\begin{array}{c}\text { Ulva lactuca } \\
\text { (Figure 1n) (C) }\end{array}$ & $\begin{array}{c}\text { Arginine, Aspartic acid, } \\
\text { Glycine }\end{array}$ & $\begin{array}{l}\text { Enhance collagen and elastin } \\
\text { synthesis }\end{array}$ & [103] \\
\hline 7 & $\begin{array}{l}\text { Porphyra umbilicalis } \\
\quad \text { (Figure 1i) (R) }\end{array}$ & $\begin{array}{l}\text { MAAs, (2:1 ratio of } \\
\text { Porphyra-334 and } \\
\text { Shinorine) }\end{array}$ & Antiaging & [104] \\
\hline 8 & $\begin{array}{c}\text { Palmaria palmata } \\
\quad \text { (Figure } 1 \mathrm{~g}), \\
\text { Catenella caespitosa (R) }\end{array}$ & MAAs & UV and UV-A protection & [105] \\
\hline 9 & $\begin{array}{c}\text { Porphyra sp., } \\
\text { Catenella caespitosa (R), Padina } \\
\text { crassa, Desmarestia aculeata }(\mathrm{P})\end{array}$ & $\begin{array}{c}\text { MAAs such as } \\
\text { Aminocyclohexenone- } \\
\text { type, } \\
\text { Aminocyclohexene } \\
\text { imine-type }\end{array}$ & $\begin{array}{l}\text { Photoprotection, Antiaging, } \\
\text { Anti-inflammatory, Antioxidant }\end{array}$ & [106] \\
\hline 10 & $\begin{array}{l}\text { Curdiea racovitzae, Iridaea cordata } \\
\text { (R) }\end{array}$ & Palythine, asterina-330 & Antioxidant, Anti-UV, Antiaging & [107] \\
\hline 11 & Porphyra sp. (R) & $\begin{array}{l}\text { Protein and } \\
\text { hydrolysates }\end{array}$ & $\begin{array}{c}\text { Moisture retention capacity and } \\
\text { viscosifying agent }\end{array}$ & {$[108,109]$} \\
\hline 12 & Palmaria sp., Porphyra sp. (R) & $\begin{array}{c}\text { High amounts of } \\
\text { Glycine and Arginine }\end{array}$ & Natural moisturizing factor & [110] \\
\hline 13 & $\begin{array}{l}\text { Chondrus crispus (Figure } 4 \mathrm{~b} \text { ), } \\
\text { Mastocarpus stellatus (Figure } 4 \mathrm{e}), \\
\text { Palmaria palmata (Figure 1g) (R) }\end{array}$ & $\begin{array}{l}\text { Palythine, Usujirene, } \\
\text { Porphyra-334, } \\
\text { Shinorine, Asterina, } \\
\text { palythinol }\end{array}$ & Antioxidant, Anti-proliferation & [111] \\
\hline 14 & $\begin{array}{l}\text { Pelvetia canaliculata } \\
\quad(\text { Figure } 4 \mathrm{~d})(\mathrm{P})\end{array}$ & Amino acids & $\begin{array}{l}\text { Antioxidant, Collagen synthesis, } \\
\text { Proteoglycan synthesis } \\
\text { stimulation }\end{array}$ & [112] \\
\hline 15 & Laminaria digitata (Figure 1f) $(\mathrm{P})$ & Proteins & Lipolytic & [113] \\
\hline 16 & Neopyropia yezoensis (R) & Peptide PPy1 & Anti-inflammatory & [114] \\
\hline 17 & $\begin{array}{l}\text { Palmaria palmata } \\
\text { (Figure } 1 \mathrm{~g})(\mathrm{R})\end{array}$ & MAAs & UV protector & [115] \\
\hline
\end{tabular}


Table 2. Cont.

\begin{tabular}{|c|c|c|c|c|}
\hline No. & Name of Macroalgae & Polysaccharides & Cosmetic Benefits & References \\
\hline 18 & Sargassum polycystum (P) & $\begin{array}{l}\text { Amino acids and } \\
\text { amines }\end{array}$ & $\begin{array}{l}\text { Anti-melanogenic or skin } \\
\text { whitening effect }\end{array}$ & [116-118] \\
\hline 19 & $\begin{array}{l}\text { Porphyra umbilicalis } \\
\quad \text { (Figure 1i) (R) }\end{array}$ & $\begin{array}{l}\text { Porphyra-334, } \\
\text { Shinorine }\end{array}$ & $\begin{array}{c}\text { Moisturization, Skin protector, } \\
\text { Antiwrinkle, Protect against } \\
\text { roughness }\end{array}$ & [119] \\
\hline 21 & Porphyra yezoensis f. coreana (R) & $\begin{array}{l}\text { Peptides, PYP1-5, } \\
\text { porphyra-334 }\end{array}$ & $\begin{array}{c}\text { Enhance Elastin and collagen } \\
\text { formation, reduce MMP } \\
\text { expression }\end{array}$ & [119] \\
\hline 22 & $\begin{array}{l}\text { Palmaria palmata (Figure 1g), } \\
\text { Porphyra umbilicalis } \\
\text { (Figure } 1 \mathrm{i})(\mathrm{R})\end{array}$ & MAAs & $\begin{array}{l}\text { Antiaging, Collagenase } \\
\text { inhibition }\end{array}$ & {$[120,121]$} \\
\hline
\end{tabular}

C, Chlorophyta; R, Rhodophyta; P, Phaeophyceae.

A natural moisturizing factor, arginine has been reported in Rhodophyta Palmaria sp. and Porphyra sp. and can be used in cosmetic products [76]. Seaweed-derived mycosporine amino acids play a role in potential photo protector against UV radiation damage, in radical scavenging, and in DNA repair systems. Hence, it can be applicable in UV protection and antioxidant activity in cosmeceutical applications [122]. Due to their selective affinity to the target cells and their capacity to change physiological roles in the skin drawn attention to skincare products. Ryu et al. [119] found increased elastin and collagen production as well as decreased the expression of matrix metalloprotein (MMP) from Neopyropia yezoensis (formerly Porphyra yezoensis) (Rhodophyta) derived peptides such as PYP1-5 and porphyra334. In addition, PYP1-5 increases expression of TIMP-1,2 and TGF-b1 protein expression, which induced collagen synthesis [123]. Mycosporine amino acids from different species of Gracilaria genus (G. tenuistipitata, G. domingensis, G. birdiae) (Rhodophyta) exhibited photoprotection activity [124]. It can also absorb ultraviolet light and also played its role as a photoprotector $[125,126]$. As compared to synthetic sunscreens, Porphyra-334 obtained from the P. umbilicalis (Figure 1i) (Rhodophyta) lower the intracellular radical oxygen species increased by UVA damage and also conquered the expression of MMP complexes which proved a better UV filter [127]. Suh et al. [128] revealed the role of MAAs in antioxidant, antiaging, and anti-inflammatory activities by ROS scavenging potential, by increasing UV suppressed genes expression, and by reducing COX-2 and involucrin expression in HaCaT cells. Red algae P. umbilicalis contain MAAs and hence act as photoprotective [128]. Other examples include Dixoniella grisea (formerly Rhodella reticulata) (Rhodophyta), Ecklonia cava and Undaria pinnatifida (Figure 1b) (Phaeophyceae) have been reported for antioxidant activity [129-132]. Some algae such as Jania rubens (Figure 1j) and Meristotheca dakarensis (Rhodophyta) that produce glycosaminoglycans, type 1 and type 3 collagen synthesis, and keratin have been widely utilized for their antiaging benefit [133]. Subramaniyan et al. [134] and Kim et al. [123] reported Neopyropia yezoensis (formerly Pyropia yezoensis) (Rhodophyta) derived peptides in the stimulation of collagen synthesis in antiaging activity (by enhancing expression of TGF Beta1 protein), elastin synthesis, and by suppressing the expression of MMP-1 protein. 

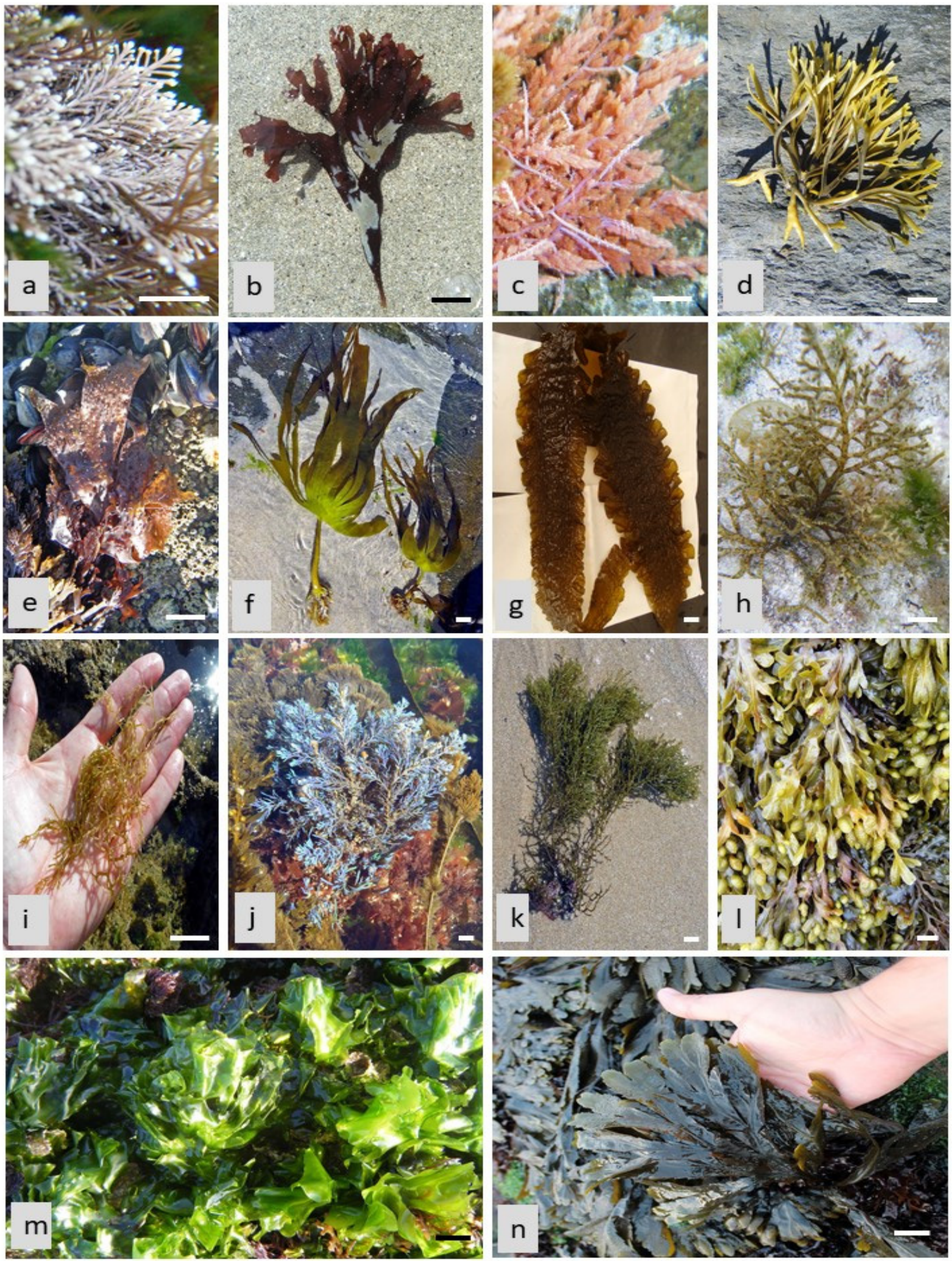

Figure 4. Seaweed species images: (a)—Corallina officinalis (R); (b) - Chondrus crispus (R); (c)_Asparagopsis armata (R); (d)-Pelvetia canaliculata (P); (e)-Mastocarpus stellatus (R); (f)-Laminaria ochroleuca (P); (g)-Saccharina latissima (P); (h)-Cystoseira compressa $(\mathrm{P})$; (i) - Gongolaria nodicaulis (P); (j) -Ericaria selaginoides (P); (k)-Gongolaria usneoides (P); (1)_Fucus spiralis (P); (m)_Ulva rigida (C); (n)_Fucus serratus (P); C-Chlorophyta; $\mathrm{R}$-Rhodophyta; $\mathrm{P}$-Phaeophyceae; Scale $=1 \mathrm{~cm}$. 


\section{Pigments}

Marine macroalgae have a broad diversity of photosynthetic pigments that capture light for the photosynthesis process. Chlorophyta (green algae) contain chlorophyll $a$, chlorophyll $b$, and carotenoids; Rhodophyta (red algae) contain chlorophyll $a$, phycobilin (phycocyanin, phycoerythrin), and carotenoids (carotene, lutein, zeaxanthin), and Phaeophyceae (brown algae) contain chlorophyll $a$, chlorophyll $c$, fucoxanthin, and different carotenoid pigments. Different macroalgae-derived pigments and cosmetic applications are reported in Table 3. These pigments provide a shield to the skin cells against harmful UV radiations [135].

Hama et al. [136] reported cosmetic formulation containing astaxanthin exhibit potential antioxidant and photoprotection effect against the cell damage caused by UV B. Marine macroalgae are widely known for different ranges of pigments; chlorophylls, carotenoids, and phycobilin are the three major classes of pigments. Carotenes, fucoxanthin, xanthophylls, and peridinin belong to carotenoids, whereas phycocyanin and phycoerythrin belong to phycobilin pigment.

Pereira et al. $[137,138]$ suggested the biological role of pigments in photoprotection as well as an antioxidant activity through the elimination of ROS. Carotenoids used as natural dyes have been reported to benefits such as antioxidant, anti-inflammatory, and radical sequestering properties [139]. Carotenoids have pro-vitamin A activity that protects the healthy skin from damaging effects as well as photooxidation from UV light [140]. Astaxanthin has various skin cosmetic benefits such as antiinflammation and antioxidants in photodamage [141]. Peng et al. [142] and D' Orazio et al. [143] showed the main biological benefits of fucoxanthin such as antitumor, anti-inflammatory, antitumor, antiobesity, and antiangiogenic activities. Carotenoids such as alpha-carotene, beta carotene, and lycopene are categorized as carotenes whereas astaxanthin, fucoxanthin, lutein, and zeaxanthin are categorized as xanthophylls. These pigments have skin benefits such as anti-inflammatory and antioxidant properties, and are useful as natural dyes. Mularczyk et al. [144] reported the antioxidative, anti-inflammation, and immunomodulatory activity of astaxanthin and suggested it as an interesting candidate for the cosmetic industry. Rphycoerythrin is commonly found in different algae such as Polysiphonia stricta, Palmaria palmata (Figure 1g), Gracilaria gracilis (Figure 1k), Gracilaria tikvahiae, and Neopyropia yezoensis (Rhodophyta). This R-phycoerythrin together with phycobiliproteins can be used as a natural color enhancer as well as in cosmetic formulations.

Cardoso et al. [145], Kim et al. [146], and Sekar and Chandramohan [147] reported the role of pigments such as phycocyanin and phycoerythrin in daily routine cosmetic products such as lipsticks, eyeliner, as well as photoprotection, antimicrobial, anti-inflammatory properties. The chemical structures of pigments found in marine macroalgae are presented in Figure 5.

Table 3. Applications of macroalgae derived pigments in skin cosmetics.

\begin{tabular}{cccc}
\hline No. & Name of Macroalgae & Pigment & Cosmetic Benefits \\
\hline 1 & Sargassum spp. & $\begin{array}{c}\text { Carotenoids, } \\
\text { Astaxanthin, } \\
\text { Beta-carotene, } \\
\text { Fucoxanthin }\end{array}$ & $\begin{array}{c}\text { Anticellulite, Antiaging, } \\
\text { Antiphotoaging, antioxidant, } \\
\text { antiviral }\end{array}$ \\
\hline 2 & Saccharina japonica $(\mathrm{P})$ & Fucoxanthin & $\begin{array}{c}\text { Inhibition of tyrosinase and } \\
\text { Melanogenesis in UVB } \\
\text { irradiated }\end{array}$ \\
\hline 3 & Cladosiphon okamuranus $(\mathrm{P})$ & Fucoxanthin & Antioxidant, DPPH inhibition \\
\hline 4 & Neopyropia yezoensis ${ }^{\circledR}$ & Phycoerythrin & Antioxidant, Anticancer, \\
Antiinflammatory
\end{tabular}


Table 3. Cont.

\begin{tabular}{|c|c|c|c|c|}
\hline No. & Name of Macroalgae & Pigment & Cosmetic Benefits & References \\
\hline 5 & $\begin{array}{l}\text { Gracilaria gracilis, Porpyridium sp. } \\
\text { (R) }\end{array}$ & $\begin{array}{l}\text { Phycobiliprotein } \\
\text { pigment such as } \\
\text { R-phycoerythrin, } \\
\text { Phycocyanin, } \\
\text { Allophycocyanins }\end{array}$ & $\begin{array}{l}\text { Antioxidant, Skin whitening } \\
\text { activity by Antimelanogenic } \\
\text { activity }\end{array}$ & [151] \\
\hline 6 & Cladophora glomera ${ }^{\circledR}(\mathrm{C})$ & $\begin{array}{l}\text { Chlorophyll a, } \\
\text { Chlorophyll b, } \\
\text { Chlorophyll c, } \\
\text { Chlorophyll d }\end{array}$ & $\begin{array}{l}\text { Antibacterial, Antioxidant, } \\
\text { Colorants, Deodorizer }\end{array}$ & [152-154] \\
\hline 7 & Portieri $^{\circledR}$ p. (R) & $\begin{array}{l}\text { Phycobiliproteins, } \\
\text { Phycoerythrin, } \\
\text { Phycocyanin }\end{array}$ & $\begin{array}{l}\text { Antioxidants, anti-inflammatory, } \\
\text { Colorants, Radical scavenger }\end{array}$ & [154] \\
\hline 8 & Cladophora glomerata (C) & Chlorophyll & Tissue growth stimulators & [155] \\
\hline 9 & Neopyropia $y^{\circledR}$ ensis (R) & Porphyran & Antioxidant, Anti-inflammatory & {$[156]$} \\
\hline 10 & $U^{\circledR} \operatorname{lactuca}(\mathrm{C})$ & $\begin{array}{l}\text { Carotenoids such as } \\
\text { astaxanthin, } \\
\text { beta-carotene, } \\
\text { fucoxanthin, lutein }\end{array}$ & $\begin{array}{l}\text { Anti-inflammatory, Antiaging, } \\
\text { Tyrosinase inhibition, } \\
\text { Antioxidants, Photoprotective }\end{array}$ & [153] \\
\hline 11 & Rhodophyta (R) & Lutein & Skin whitening & {$[157]$} \\
\hline 12 & Paraglossum lancifolium (R) & $\begin{array}{l}\text { Lipid soluble pigments } \\
\text { such as Xanthophyll } \\
\text { and Carotenoids } \\
\text { Beta-carotene, Lutein }\end{array}$ & $\begin{array}{l}\text { Antioxidant, Anti-inflammatory, } \\
\text { Antiphotoaging, } \\
\text { Photoprotection, } \\
\text { Anti-photoaging }\end{array}$ & [158] \\
\hline 13 & Undaria pinnatifida $(\mathrm{P})$ & Fucoxanthin & Photoprotective & [159] \\
\hline 14 & Porphyra sp. (P) & $\begin{array}{l}\text { Zeaxanthin, Alpha and } \\
\text { beta carotene }\end{array}$ & $\begin{array}{c}\text { Anti-inflammatory, } \\
\text { Photoprotection, Antioxidant, } \\
\text { Antiaging }\end{array}$ & [160] \\
\hline 15 & Gracilaria gracilis (Figure 1k) (R) & $\begin{array}{l}\text { Phycobiliproteins } \\
\text { (R-phycoerythrin } \\
\text { allophycocyanin, } \\
\text { Phycocyanin) }\end{array}$ & Antioxidant & [151] \\
\hline 16 & Sargassum siliquastrum $(\mathrm{P})$ & Fucoxanthin & $\begin{array}{l}\text { Skin protector, Antiphotoaging, } \\
\text { Antiwrinkle }\end{array}$ & [161] \\
\hline 17 & Ulva lactuca (C) & $\begin{array}{l}\text { Zeaxanthin, } \\
\text { Neoxanthin, } \\
\text { Antheraxanthin, } \\
\text { Siphonein, } \\
\text { Siphoxanthin, }\end{array}$ & $\begin{array}{l}\text { Photoprotection, } \\
\text { Antiphotoaging, } \\
\text { Anti-inflammatory }\end{array}$ & [162] \\
\hline 18 & Himanthalia elongata $(\mathrm{P})$ & Fucoxanthin extract & Antioxidant & [163] \\
\hline 19 & Ascophyllum nodosum (P) & Fucoxanthin & Antiagin, Antiwrinkle & {$[164]$} \\
\hline 20 & Fucus vesiculosus (P) & Fucoxanthin & Antioxidant & [165] \\
\hline 21 & Phaeophyta & Fucoxanthin & Antiphotoaging & [166] \\
\hline 22 & Sargassum siliquastrum $(\mathrm{P})$ & Fucoxanthin & $\begin{array}{c}\text { Anti-melanogenic (skin } \\
\text { whitening effect), Antioxidant, } \\
\text { Anti-inflammatory }\end{array}$ & {$[167]$} \\
\hline 23 & Gelidium crinale $(\mathrm{R})$ & Carotenoids & Antioxidant & [168] \\
\hline
\end{tabular}

C, Chlorophyta; R, Rhodophyta; P, Phaeophyceae. 


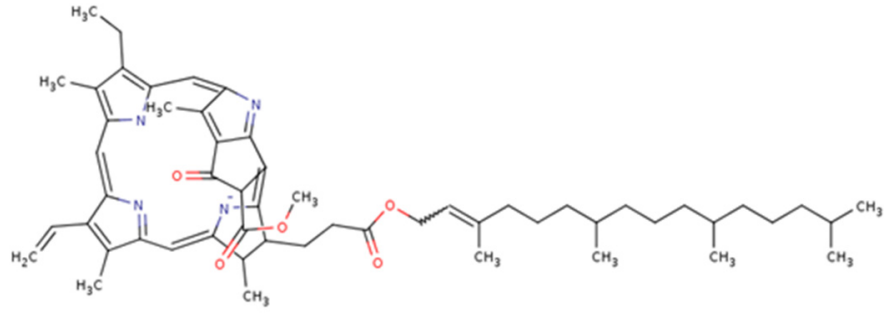

(a)

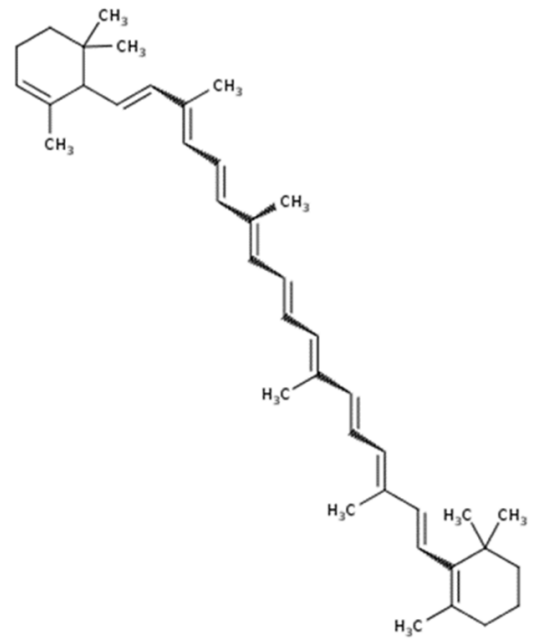

(c)

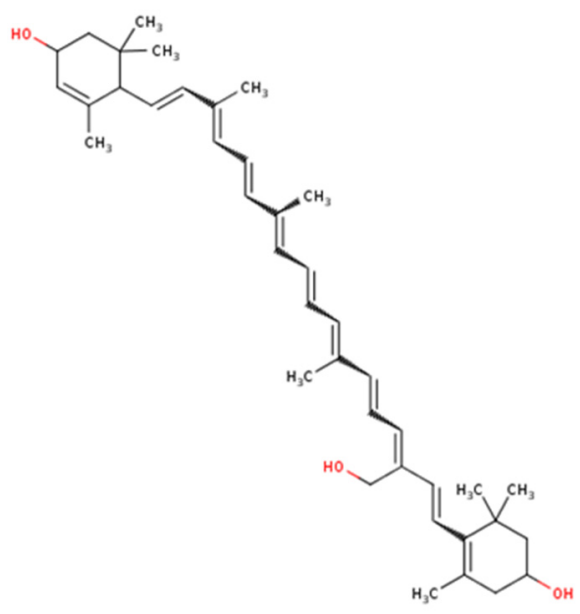

(e)

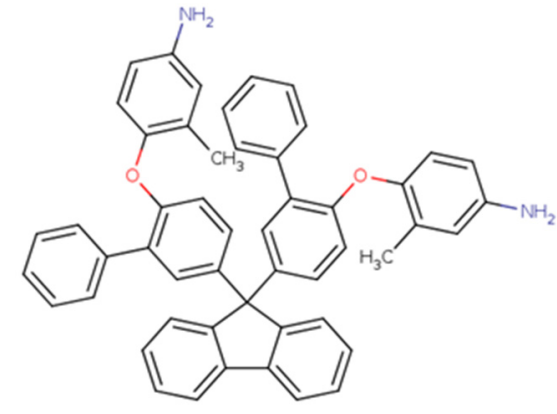

(b)

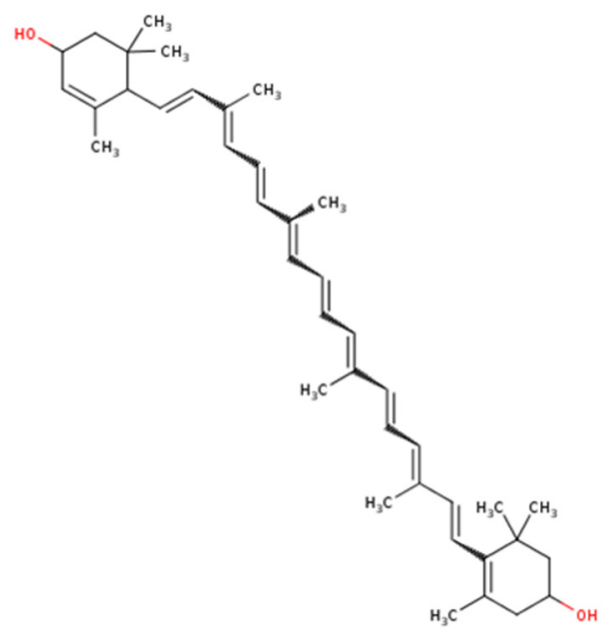

(d)

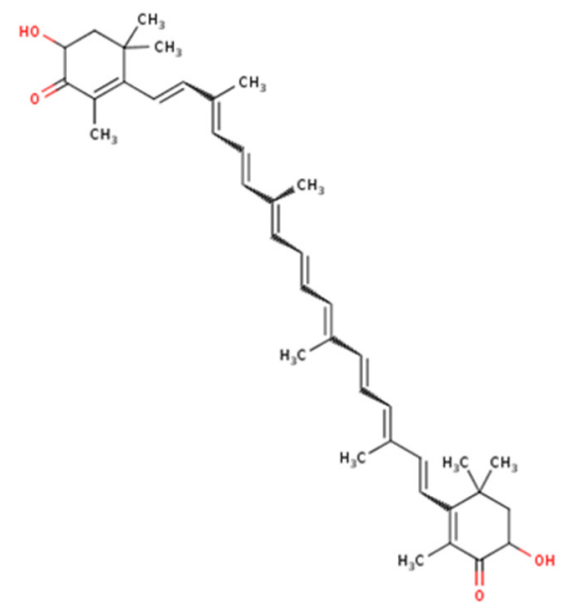

(f)

Figure 5. Cont. 


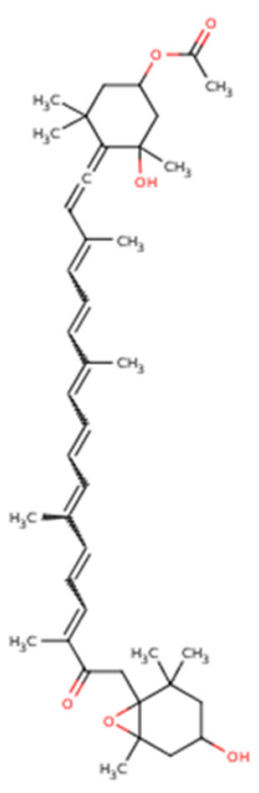

$(\mathrm{g})$

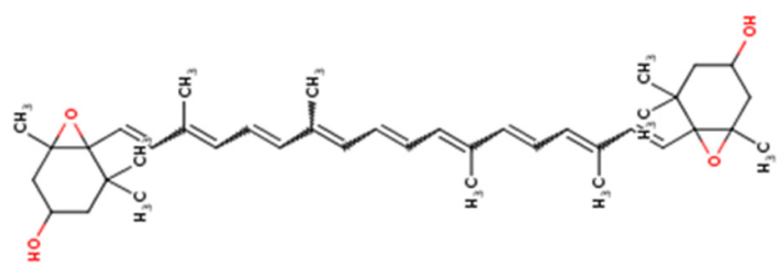

(h)

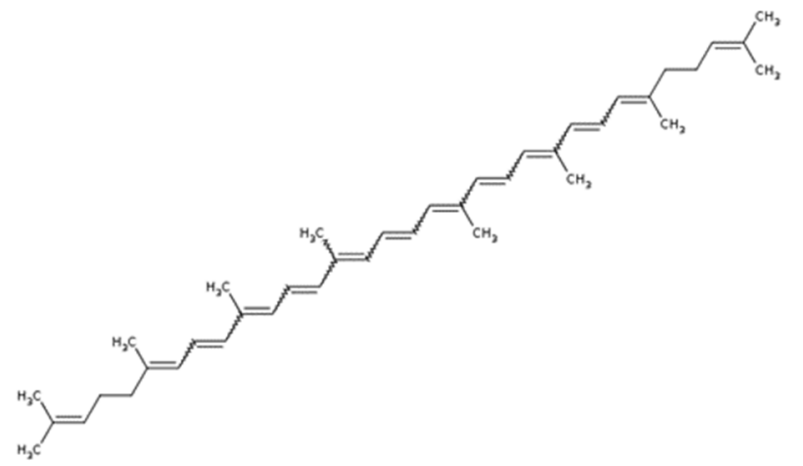

(i)

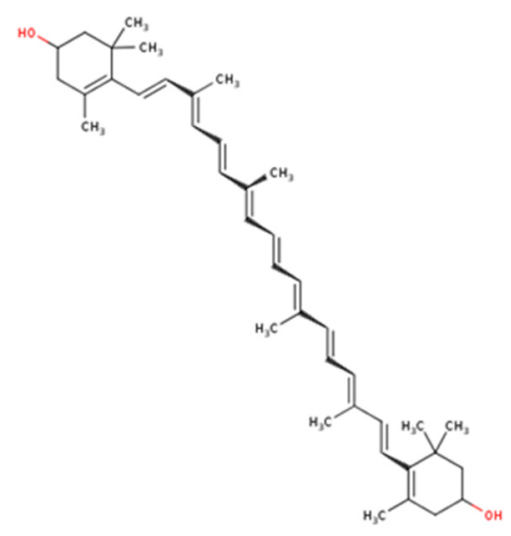

(j)

Figure 5. Chemical structures of marine macroalgae derived pigments. (a) Chlorophyll A, (b) phycoerythrine, (c) alpha-carotene, (d) xanthophyll, (e) loraxanthin, (f) astaxanthin, (g) fucoxanthin, (h) violaxanthin, (i) lycopene, and (j) zeaxanthin.

\section{Phenolic Compounds}

Phenolic compounds are one of the secondary metabolites that make an important group of components for skin cosmetic benefits. Due to wide varieties of biological actions, they can be incorporated in various skin cosmetic preparations. Theyr can be categorized into simple phenolic compounds and polyphenols, comprising bromophenols, phlorotannins, flavonoids, terpenoids, etc. [169]. Marine macroalgae0derived phenolic compounds and their cosmetic benefits are presented in Table 4. Gómez et al. [170] and Morais et al. [171] reported the presence of a high concentration of phlorotannins in brown algae whereas bromophenol, phenolic acids, terpenoids, and flavonoids are predominant in green and red macroalgae. Different phenolic groups have different cosmeceutical benefits with Phlorotannin acting as a hyaluronidase inhibitor and conferring cosmetic effects (such as antiwrinkle, antiaging, skin whitening, and antiallergic effects). Marine macroalgae are the producer of these molecules and lead to valuable ingredients in cosmetic formulations. Mateos et al. [172] revealed bioactive potentials of seaweed-derived phenolic compounds with antimicrobial, anticancer, antioxidant, anti-inflammatory properties, etc. which make it very attractive to cosmetic and cosmeceutical products. Phlorotannin has more attention 
in cosmetic applications due to multiple benefits in cosmetics such as antioxidant, antiinflammatory, anti-allergic, hyaluronidase inhibitor, and MMP (matrix metalloproteinase) inhibition [173]. In addition, it shows protection against damaging UV rays, but also gives protection against ionizing radiations [174]. Seaweed-derived Bromophenol reported organoleptic properties (such as flavor) [175]. Sanjeewa et al. [176] reported antiallergic, antiwrinkle, and antiaging activities of phlorotannins due to inhibition of hyaluronidase enzyme. Polyphenol content from the brown alga Fucus vesiculosus reported UV protection, skin-soothing benefits as well as skin age spot reduction [177]. The chemical structures of macroalgae derived phenolic compounds are illustrated in Figure 6.<smiles>Oc1cc(O)cc(Oc2c(O)cc(O)c3c2Oc2c(O)cc(O)cc2O3)c1</smiles>

(a)<smiles>Oc1ccccc1</smiles>

(c)<smiles>Oc1cc(O)cc(Oc2c(O)cc(O)c3c2Oc2c(cc4oc5c(Oc6cc(O)cc(O)c6)c(O)cc(O)c5c4c2O)O3)c1</smiles>

(d)

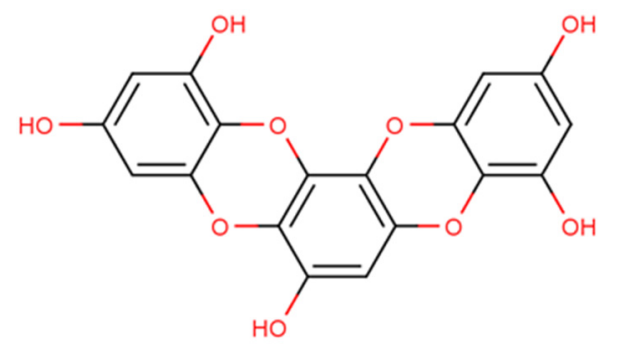

(f)<smiles>Oc1cc(O)cc(Oc2c(O)cc(O)c3c2Oc2cc(Oc4c(O)cc(Oc5c(O)cc(O)c6c5Oc5c(O)cc(O)cc5O6)cc4O)cc(O)c2O3)c1</smiles>

(b)<smiles>Oc1cc(O)cc(Oc2c(O)cc(Oc3c(O)cc(Oc4c(O)cc(Oc5cc(O)c(Oc6cc(O)c(Oc7cc(O)c(Oc8c(O)cc(O)cc8O)cc7O)c(Oc7c(O)cc(O)cc7O)c6)c(O)c5)cc4O)cc3O)cc2O)c1</smiles>

(e)<smiles>CC(C)=CC(O)C(O)C(C)=CCCC(C)=CCCC1(C)CCc2cc(O)cc(C)c2O1</smiles>

(g)

Figure 6. Chemical structures of marine macroalgae derived phenolic compounds. (a) Eckol, (b) dieckol, (c) phenol, (d) phlorofucofuroeckol B, (e) octaphlorethol A, (f) eckstolonol, (g) sargachromanol E.

Tyrosinase inhibition and skin whitening effect exhibited by phlorotannin from brown alga Sargassum fusiforme (formerly Hizikia fusiformis) [178]. Bromophenol from Laurencia sp. reported antioxidant and antimicrobial effects which can be concerned for photopro- 
tection in the cosmetic formulation [175]. Li et al. [179] and Zou et al. [180] studied the biological activity of phlorotannin from different brown marine macroalgae such as Ecklonia cava, E. cava subsp. stolonifera (formerly E. stolonifera), E. cava subsp. kurome (formerly Ecklonia kurome), Ishige okamurae, S. fusiforme, Eisenia bicyclis, U. pinnatifida (Figure 1b) and Sargassum thunbergii (Phaeophyceae). Phlorotannin is phloroglucinol-based polyphenols, mainly found in brown algae. Phlorotannins have a wide range of beneficial applications such as anti-melanogenesis, antiaging, and antioxidants which are applicable in cosmetic formulations [181-185]. In a dose-dependent manner, Ryu et al. [186] studied that methanol extract of Corallina pilulifera which is rich in phenolic content inhibited the matrix metalloproteinase 2,9 expression in UV induced dermal fibroblast cells. Joe et al. [182] and Kong et al. [187] found strong MMP inhibition by E. bicyclis, E. cava, and E. cava subsp. Stolonifera derived Phlorotannin.

Table 4. Applications of macroalgae derived phenolic compounds in skin cosmetics.

\begin{tabular}{|c|c|c|c|c|}
\hline No. & Name of Macroalgae & Phenolic Compound/s & $\begin{array}{l}\text { Characterization or Analysis of } \\
\text { Phenolic Compounds }\end{array}$ & References \\
\hline 1 & Macrocystis pyrifera $(\mathrm{P})$ & $\begin{array}{c}\text { Phlorotannins, } \\
\text { Phloroeckol, Tetrameric } \\
\text { phloroglucinol }\end{array}$ & Antioxidant, Antidiabetic, Antiaging & [188] \\
\hline 2 & $\begin{array}{l}\text { Ascophyllum nodosum } \\
\quad \text { (Figure } 10)(\mathrm{P})\end{array}$ & Ascophyllan & MMP inhibition & [189] \\
\hline 3 & Cystoseira foeniculacea $(\mathrm{P})$ & Polyphenol & Antioxidant & {$[190]$} \\
\hline 4 & Stephanocystis hakodatensis $(\mathrm{P})$ & Phenol & Antioxidant & [191] \\
\hline 5 & Ecklonia cava subsp. Stolonifera (P) & Fucofuroeckol-A & Protection against UVB radiation & [192] \\
\hline 7 & Corallina pilulifera $(\mathrm{R})$ & Phlorotannins & $\begin{array}{l}\text { Antiaging, antiinflammatio, } \\
\text { antioxidants, antiallergic, UV screens }\end{array}$ & [193] \\
\hline 8 & Ishige foliacea $(\mathrm{P})$ & Phlorotannin & $\begin{array}{l}\text { Antimelanogenic, inhibition of } \\
\text { tyrosinase and melanin synthesis }\end{array}$ & {$[194,195]$} \\
\hline 10 & $\begin{array}{l}\text { Laminaria ochroleuca } \\
\quad(\text { Figure } 4 \mathrm{f})(\mathrm{P})\end{array}$ & Polyphenol & Antioxidant & [196] \\
\hline 11 & Caulerpa racemo ${ }^{\circledR}(\mathrm{C})$ & $\begin{array}{l}\text { Flavonoids, } \\
\text { Hydroquinone, } \\
\text { Saponins }\end{array}$ & Tyrosinase inhibitor & [197] \\
\hline 12 & Ecklonia cava $(\mathrm{P})$ & Dioxinodehydroeckol & UV B protective & {$[198]$} \\
\hline 13 & Ecklonia cava subsp. stolonifera $(\mathrm{P})$ & Phlorotannins & $\begin{array}{c}\text { Inhibition of Matric metalloproteins } \\
\text { (MMPs), Antiwrinkle, Tyrosinase } \\
\text { inhibitor, Skin whitener }\end{array}$ & [199] \\
\hline 14 & Saccharina latissima (Figure 4g) (P) & Phenol & Antioxidant & [200] \\
\hline 15 & Ecklonia cava $(\mathrm{P})$ & Dieckol & Anti-adipogenesis & [201] \\
\hline 16 & Ecklonia cava subsp. kurome $(\mathrm{P})$ & Phlorotannin & $\begin{array}{c}\text { Anti-inflammatory, Hyaluronidase } \\
\text { inhibition }\end{array}$ & [202] \\
\hline 17 & Caulerp ${ }^{\circledR}$ p. (C) & Flavonoids, Phenols & Tyrosinase inhibitors & [203] \\
\hline 18 & Rhodomela conf ${ }^{\circledR}$ oides (R) & $\begin{array}{l}\text { Polyphenol, } \\
\text { Bromophenol }\end{array}$ & $\begin{array}{c}\text { Antioxidant, Antimicrobial, DPPH } \\
\text { inhibition }\end{array}$ & [204] \\
\hline 19 & $\begin{array}{c}\text { Eisenia bicyclis, Ecklonia Cava subsp. } \\
\text { stolonifera }(\mathrm{P})\end{array}$ & Eckol & $\begin{array}{l}\text { Anti-inflammation, Skin whitening } \\
\text { activity }\end{array}$ & {$[205,206]$} \\
\hline 20 & Schizymenia dubyi (Figure 1c) (R) & Phenol & $\begin{array}{c}\text { Anti-melanogenic, Tyrosinase } \\
\text { inhibition }\end{array}$ & [207] \\
\hline
\end{tabular}


Table 4. Cont.

\begin{tabular}{|c|c|c|c|c|}
\hline No. & Name of Macroalgae & Phenolic Compound/s & $\begin{array}{l}\text { Characterization or Analysis of } \\
\text { Phenolic Compounds }\end{array}$ & References \\
\hline \multirow[t]{2}{*}{21} & $\begin{array}{l}\text { Cystoseira compressa } \\
\text { (Figure } 4 \mathrm{~h})(\mathrm{P})\end{array}$ & Fuhalol & Antioxidant & [208] \\
\hline & $\begin{array}{l}\text { Cystoseira compressa } \\
\text { (Figure } 4 \mathrm{~h})(\mathrm{P})\end{array}$ & Fuhalol & Antioxidant & [208] \\
\hline 22 & Ecklonia cava $(\mathrm{P})$ & dieckol & Promotes hair growth & [209] \\
\hline 23 & $\begin{array}{c}\text { Fucus vesiculosus (Figure 1a), } \\
\text { Gongolaria nodicaulis (Figure 4i), } \\
\text { Ericaria selaginoides (Figure 4j), } \\
\text { Gongolaria usneoides (Figure 4k), } \\
\text { Ecklonia cava (P) }\end{array}$ & $\begin{array}{l}\text { Phlorotannins such as } \\
\text { Fucophloroethol, } \\
\text { Fucodiphloroethol, } \\
\text { Fucotripholoroethol, } \\
\text { Phlorofucofuroeckol } \\
\text { bieckol or dieckol }\end{array}$ & $\begin{array}{l}\text { Skin whitening effect, Antioxidant, } \\
\text { Anti-inflammatory, Antihistamine, } \\
\text { Photoprotection }\end{array}$ & [210] \\
\hline 24 & $\begin{array}{l}\text { Ascophyllum nodosum } \\
\quad \text { (Figure 1o) }(\mathrm{P})\end{array}$ & $\begin{array}{l}\text { Phlorotannins, Eckols, } \\
\text { Fucols, Phlorethols }\end{array}$ & $\begin{array}{c}\text { Inhibition of tyrosinase, } \\
\text { Anti-inflammation, Anti UV, } \\
\text { Anti-allergic, Chelators, Antiaging, } \\
\text { Hyaluronidase inhibitor }\end{array}$ & [210] \\
\hline 25 & Meristotheca dakarensis (R) & Glucosaminoglycan & Anti-aging, Collagen synthesis & [13] \\
\hline 26 & $\begin{array}{c}\text { Gongolaria nodicaulis, } \\
\text { Ericaria selaginoides, } \\
\text { Gongolaria usneoides (Figure 4k) (P) }\end{array}$ & $\begin{array}{l}\text { Phlorotannins such as } \\
\text { bioeckol, 7-phloroeckol, } \\
\text { phlorofucofuroeckol, } \\
\text { fucophloroethol }\end{array}$ & $\begin{array}{l}\text { Anti-inflammation, Antioxidant, } \\
\text { Anti-aging, Inhibition of } \\
\text { hyaluronidase }\end{array}$ & [210] \\
\hline 27 & $\begin{array}{l}\text { Fucus spiralis } \\
\text { (Figure } 41)(\mathrm{P})\end{array}$ & Phlorotannins & $\begin{array}{l}\text { Inhibition of lipid peroxidation, } \\
\text { hyaluronidase inhibitor, antiaging, } \\
\text { antiwrinkle, Anti-inflammatory, } \\
\text { Antiwrinkle }\end{array}$ & [210] \\
\hline 28 & $\begin{array}{c}\text { Ecklonia cava, Ecklonia cava subsp. } \\
\text { stolonifera }(\mathrm{P})\end{array}$ & $\begin{array}{c}\text { Eckol, 6,6'-bieckol, } \\
\text { doeckol, } \\
\text { Phlorofucofuroeckol-A, } \\
\text { 8,8'-bieckol }\end{array}$ & Anti-allergic & [211] \\
\hline 29 & $\begin{array}{l}\text { Eisenia bicyclis, Ecklonia cava subsp. } \\
\text { stolonifera }\end{array}$ & Phlorofucofuroeckol A & Hepatoprotective, Anti-tyrosinase & {$[212,213]$} \\
\hline 30 & Eisenia arborea, Ecklonia bicyclis (P) & Phlorotannins & $\begin{array}{l}\text { Anti-inflammation, Hyaluronidase } \\
\text { inhibitor, antiwrinkle }\end{array}$ & [214] \\
\hline 31 & Eisenia arborea $(\mathrm{P})$ & Phlorofucofuroeckol A & Anti-allergic & [215] \\
\hline 32 & $\begin{array}{c}\text { Ascophyllum nodosum } \\
\text { (Figure 1o), } \\
\text { Fucus serratus (Figure } 4 \mathrm{n}), \\
\text { Himanthalia elongata (Figure } 1 \mathrm{~h}), \\
\text { Sargassum muticum }(\mathrm{P})\end{array}$ & Phlorotannins & $\begin{array}{l}\text { Antioxidant, Antibacterial, antiviral, } \\
\text { photoprotection, Anti-inflammatory }\end{array}$ & [216-218] \\
\hline 33 & Ecklonia cava $(\mathrm{P})$ & $\begin{array}{l}\text { Eckols, fucols, } \\
\text { phlorethols, Fuhalols, } \\
\text { fucophlorethol }\end{array}$ & $\begin{array}{c}\text { Anti-aging, Anti-inflammation, } \\
\text { Hyaluronidase inhibitor, antiallergic, } \\
\text { UV protector }\end{array}$ & [218] \\
\hline
\end{tabular}

C, Chlorophyta; R, Rhodophyta; P, Phaeophyceae.

\section{Fatty Acids}

Seaweeds are well known for various types of fatty acids such as glycolipids, triglycerides, sterols, and phospholipids. The chemical structures of marine algae derived fatty acids are illustrated in Figure 7. These have been reported as being higher in seaweed as compared to terrestrial plants. Different types of fatty acids from different macroalgae and its cosmetic benefits are presented in Table 5. Dawczynski et al. [219] reported antiallergic and antiinflammation activity and skin protector (emollient) of seaweed-derived fatty acids. 
Rhimou et al. [220] studied effective bacterial growth inhibition from methanolic extract of Ceramium virgatum (formerly Ceramium rubrum) (Figure 11) (Rhodophyta) against Escherichia coli, Enterococcus faecalis, and Staphylococcus aureus. Although, macroalgaederived fatty acids (SFA, MUFA, PUFA) have been characterized for their antioxidant, anti-inflammatory, anti-allergic activities, etc. It may serve as soothing benefit that makes skin safer by maintaining hydration. The presence of polyunsaturated fatty acids (omega-3, omega-6) is responsible for skin nutrition and for maintaining skin health. These fatty acids are important regulators of several cellular processes as well as good health mediators [221]. Pimentel et al. [222] and Kumari et al. [223] suggested a predominant amount of eicosapentaenoic acid (EPA) and arachidonic acid in Phaeophyta and Rhodophyta whereas Chlorophyta have a high amount of docosahexaenoic acid (DHA). Higher quantities of C18-PUFAs presented in Chlorophyta species while C20-PUFAs in Rhodophyta species. Antiaging and antioxidant activity was reported in methanolic extract of Corallina pilulifera (Rhodophyta) by reducing the expression of gelatinase and by inhibition of free radical oxidation in human dermal fibroblast and human fibrosarcoma (HT-1080) [186]. A previous study reported that topical applications of medium-chain triglycerides, linoleic acid, soy lecithin, and vitamin A can promote wound healing in mice. It plays its role in the mediation of inflammation, cell proliferation, wound contraction, and tissue remodeling [224]. The fatty acids, mainly mixtures of triglycerides of saturated and unsaturated fatty acids commonly used as an ingredient in cosmetic formulation. Bonnet, [225], Bialek et al. [226], Rabasco and González et al. [227], and Zielinska and Nowak [228] suggested the significant importance of fatty acids in healing effects, which can be used in creams, emulsions, cosmetic masks, lipsticks, bath fluids, nail polishes, etc. Moreover, fatty acids play an important in overcoming excessive dryness of the skin, and their use is beneficial for skin and hair.

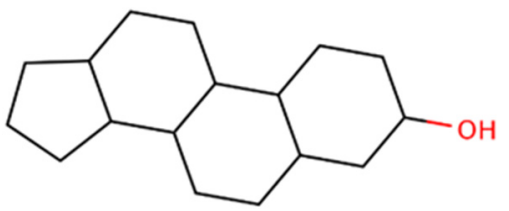

(a)<smiles>CC(C)C(C)CCC(C)C1CCC2C3CC=C4CC(O)CCC4(C)C3CCC12C</smiles>

(c)

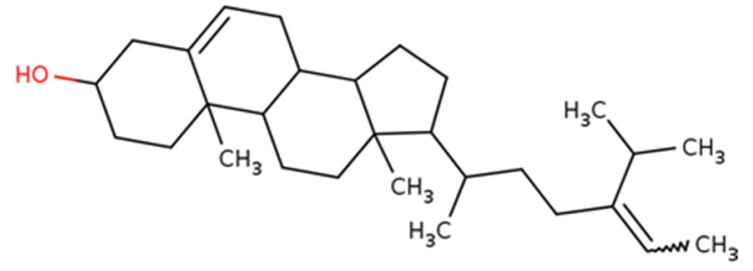

(b)

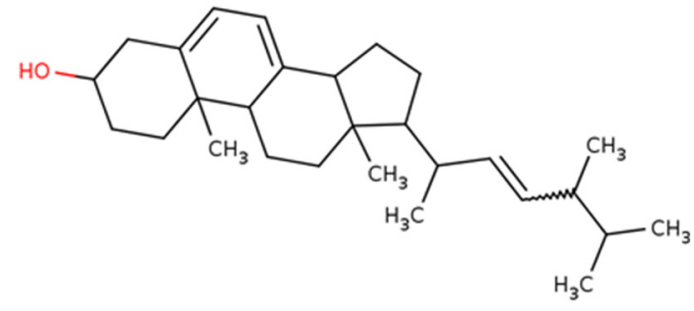

(d)

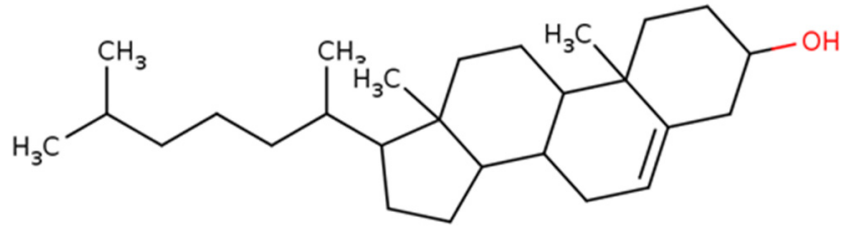

(e)

Figure 7. Chemical structures of marine macroalgae derived fatty acids. (a) Sterol, (b) fucosterol, (c) campesterol, (d) ergosterol, (e) cholesterol. 
Table 5. Applications of macroalgae derived lipids and fatty acids in skin cosmetics.

\begin{tabular}{|c|c|c|c|c|}
\hline No. & Name of Macroalgae & Fatty acid & Cosmetic Benefits & References \\
\hline 1 & $\begin{array}{l}\text { Chondrus crispus } \\
\text { (Figure } 4 \mathrm{~b})(\mathrm{R})\end{array}$ & $\begin{array}{l}\text { EPA, AA, DHA, GLA, LA, } \\
\text { Palmitic acid, Oleic acid }\end{array}$ & $\begin{array}{l}\text { Antiallergic, Anti-aging, } \\
\text { Anti-inflammation, Antiwrinkle, } \\
\text { Antimicrobial, Emollients, }\end{array}$ & [229] \\
\hline 2 & $\begin{array}{l}\text { Undaria pinnatifida } \\
\quad \text { (Figure } 1 \mathrm{~b})(\mathrm{P})\end{array}$ & PUFA & Anti-inflammatory & [229] \\
\hline 3 & $\begin{array}{c}\text { Ulva lactuca } \\
\text { (Figure 1n) (P) }\end{array}$ & $\begin{array}{l}\text { Fatty acid such as C18 and } \\
\text { C16 type }\end{array}$ & $\begin{array}{l}\text { In-vitro and in-vivo Nrf2-ARE } \\
\text { activation, Cell protective, } \\
\text { Antioxidant }\end{array}$ & [230] \\
\hline 4 & $\begin{array}{l}\text { Phaeophyceae } \\
\text { (Brown algae) }(\mathrm{P})\end{array}$ & Unsaturated Fatty acids & Antioxidant & [231] \\
\hline 5 & $\begin{array}{c}\text { Ulva lactuca } \\
\text { (Figure 1n) (P) }\end{array}$ & Lipopeptides & $\begin{array}{l}\text { Inhibition of elastase, enhance } \\
\text { collagen synthesis }\end{array}$ & [232] \\
\hline 6 & $\begin{array}{l}\text { Himanthalia elongata } \\
\quad \text { (Figure } 1 \mathrm{~h})(\mathrm{P})\end{array}$ & $\begin{array}{l}\text { Fatty acids and volatile } \\
\text { compounds }\end{array}$ & Antioxidant, Antimicrobial & [233] \\
\hline 7 & Porphyridium purpureum (R) & $\begin{array}{c}\text { Eicosapentaenoic acid, } \\
\text { Docosahexaenoic acid, } \\
\text { Eicosatetraenoic acid, } \\
\text { Polyunsaturated omega-3 } \\
\text { fatty acids }\end{array}$ & $\begin{array}{l}\text { Antioxidant, Anti-inflammatory, } \\
\text { Anti-photoaging }\end{array}$ & [234] \\
\hline 8 & $\begin{array}{c}\text { Ulva rigida (Figure } 4 \mathrm{~m})(\mathrm{C}) \\
\text { Gracilaria sp. }(\mathrm{R}), \text { Fucus vesiculosus } \\
\text { (Figure } 1 \mathrm{a}), \text { Saccharina latissima } \\
\text { (Figure } 4 \mathrm{~g})(\mathrm{P})\end{array}$ & Lipidic profile & Antioxidant & [235] \\
\hline 9 & Sargassum fusiforme $(\mathrm{P})$ & Fucosterol & $\begin{array}{l}\text { Protection against photodamage, UVB } \\
\text { protector, MMP inhibition, Enhance } \\
\text { procollagen formation, } \\
\text { Anti-inflammatory }\end{array}$ & {$[236,237]$} \\
\hline 10 & $\begin{array}{l}\text { Gracilariopsis longissima }(\mathrm{R}), \\
\quad \text { Saccharina japonica }(\mathrm{P})\end{array}$ & $\begin{array}{c}\text { (8E)-10-oxo-8-octadecenoic } \\
\text { acid, } \\
\text { (E)-9-oxo-10-octadecenoic } \\
\text { acid, Myristic acid, } \\
\text { Palmitic acid }\end{array}$ & Anti-inflammatory & [238] \\
\hline 11 & Silvetia siliquosa $(\mathrm{P})$ & Fucosterol & $\begin{array}{l}\text { Antioxidant, Stimulate antioxidant } \\
\text { enzymes such as catalase, glutathione } \\
\text { peroxidase }\end{array}$ & {$[239,240]$} \\
\hline 14 & Sargassum fusiforme (P) & Fucosterol & Anti-aging, MMP inhibition & [241] \\
\hline 15 & Codium fragile $(\mathrm{C})$ & Sterol & Anti-inflammatory & [242] \\
\hline
\end{tabular}

C, Chlorophyta; R, Rhodophyta; P, Phaeophyceae.

\section{Minerals}

Depending on the environment in which macroalgae inhabit, they are highly diversified in mineral composition (especially with regards to trace elements including zinc, magnesium, aluminum, silica, copper, iodine, selenium, iron, manganese, and micronutrients including calcium, sodium, phosphorus, potassium, and chlorine). Kuda et al. [243] examined the mineral content in concentrates of harvested seaweed Sargassum coreanum (formerly Sargassum ringgoldianum subsp. coreanum) from Japanese beaches. They found the highest concentration of potassium, magnesium, and calcium in these samples. Likewise, Kappaphycus alvarezii (Figure 1m) (Rhodophyta) presented high levels of magnesium and calcium ions [244].

Minerals have a very essential vital role as cofactors of different metalloenzymes [245]. Moreover, a combination of calcium and magnesium improves barrier repairs in topical 
skincare products [246]. Indeed, acid-induced burns are relieved by gel solution containing calcium gluconate solution [247]. Likewise, magnesium silicate (talc) and magnesium sulphate (i.e., Epsom salts) have reported enhancement of skin benefits. Talc is most frequently useful in baby skin powders to prevent diaper rash. In adults, it can be used as a lubricant and to reduce wetness in the perineal and axillary areas [248]. In addition, Boisseau et al. [249] found improvements in skin softness and exfoliation, relief in muscle tension, and the promotion of relaxation by Epsom salts. They also reported the key regulatory role of $\mathrm{Mg}^{++}$and $\mathrm{Ca}^{++}$in the proliferation and differentiation of keratinocytes. Likewise, magnesium silicate (talc) and magnesium sulphate (Epsom salts) have reported enhancement of skin benefits.

Talc is most frequently useful in baby skin powders to prevent diaper rash as well as in adults to reduce wetness in the perineal and axilla areas (and as a lubricant) [250]. They also reported the key regulatory role of $\mathrm{Mg}^{++}$and $\mathrm{Ca}^{++}$in the proliferation and differentiation of keratinocytes. ZnO-based skin protectants are cost-effective, easily formulated, and stable under aerobic conditions $[250,251]$. Zinc oxide is superior to zinc sulphate to mitigate inflammation and enhance re-epithelization of partial-thickness porcine skin [252]. Due to low water solubility, it sustains in the skin at the wound site. Newman et al. [253] revealed the importance of skin in sunburned skin and under ultraviolet exposure. Bissett et al. [254] found significantly delayed UV-induced tumors in Guinea pigs and mouse models by topical use of a 2-furildioxime (iron chelator).

Borkow et al. [255] exhibited a significant role of copper oxide in reducing facial wrinkles and an improvement of skin appearance. Besides, selenium is useful to protect the skin by involving antioxidant enzymes, especially glutathione, peroxidase, and thioredoxin reductase [256]. Selenium mitigates the effect of UV exposure as well as inhibits photodamage and may reverse photoaging [257-259]. An application of culture media containing selenium or selenomethionine significantly reduced UV-induced death of keratinocytes and melanocytes [260].

\section{Challenges and Opportunities}

There are more than 1000 substances derived from natural sources enlisted in the INCI (International Nomenclature of Cosmetic Ingredients), of which approx. 300 substances were considered harmful to the skin and the environment and about 50 considered to have carcinogenic properties. No formulations to be utilized in skin care is $100 \%$ harmless [261]. However, users have changed their choice and become highly dependent on green products to overcome the effects of synthetic cosmetic ingredients. Macroalgae have received much attentiveness in investigating sustainable bioactive ingredients that are secure, effective, and natural [262]. A variety of marine macroalgae-derived biological active components has been given more significant values in preparing formulation due to their huge benefits on the skin $[263,264]$. Macroalgae are found to be safer as they have very insignificant cytotoxicity when analysed on human cell lines and can be used to extract bioactive components by using novel extraction techniques $[265,266]$. Marine algae are widely utilized in biofuel production, wastewater treatment, food, and agriculture sector (as well as the pharmaceutical sector) [267]. By product of these industries could also be a promising compound for skin applications in an effectual and cost-efficient alternative to synthetic ones [268]. There are many challenges in the application of macroalgae as a cosmeceutical component such as culturing, harvesting, extraction, characterization, etc. Cultivation of macroalgae on large scale requires optimum conditions such as the light of a particular wavelength, $\mathrm{pH}$, sterilization, nutrients availability, amount of $\mathrm{CO}_{2}$, temperature, salinity, carbon source, and nutrients [269]. According to the species of macroalgae and its application, the type and design of biological reactor used for cultivation. Mainly macroalgae collected from the sea or by cultivating in the sea environment in a specific season. Compared to that, culturing in a lab-based bioreactor is expensive and it gets contamination easily. Macroalgae are easily cultivated by using seawater instead of freshwater, which supplies nutrients, reduces cost, and improves the production of 
seaweed-based bioactive ingredients such as lipids carbohydrates, proteins, amino acids, and phenolic compounds [269]. Nevertheless, sea cultivation could be a challenge as well as needs large amount of seawater, and the cost of construction, well-functioning, and up keeping of the space are substantial. Sometimes, open culturing may affect algae culture and low quality of yield which was affected by the biotic and abiotic factors [270].

The extraction procedure of bioactive ingredients can be classified into conventional and novel techniques. Some are routinely used methods such as Soxhlet, soaking, boiling, saponification, classical solvent-based procedures, and maceration, whereas others are novel methods including extraction using enzymes, microwave digestion, ultrasound, or supercritical fluid-based methods. The novel extraction procedure is preferred as it offers several benefits including high yield, high quality, minimum usage of solvents, and low time requirement. The enzyme-based method is a safe and environment-friendly approach for macroalgae-derived metabolites. By using various enzymes, different types of algae-based metabolites can be extracted. This procedure can also reduce the problem of water dissolution, immiscibility of biologically active components, and maintain the efficacy and high catalytic efficiency of phycocompounds [266,271]. Microwave-assisted extraction in the open vessel is safe, effective, and useful for bigger samples. It also reduces the extraction time and minimizes solvent utilization [272,273]. Sometimes, high power, high temperature, and high amount of pressure may degrade the yield and reduce its amount. Moreover, low pressure exhibited its effect on the product of polysaccharides from the cells. Additionally, an increase in time can directly increase the product but increasing time also causes degradation of polysaccharides [274]. Due to its non-toxicity and low cost, carbon dioxide is often used in supercritical fluid-based extraction methods. It is beneficial to extract non-polar compounds such as fatty acids, phenolic, sterols, phenolic compounds, and some pigments [274]. Ethanol solvent has been applicable as a cosolvent to improve the efficacy of phenolic components and carotenoids in this method [275]. Likewise, ultrasound-based extraction is used to extract phenolic ingredients, but this may be influenced by time, temperature, power, and the intensity of the ultrasound. Sometimes, the optimum extraction time is also required to stop the metabolites degradation. It is mainly useful in the extraction of phenolic compounds and laminarin. Increasing the efficiency and yield of phycobiliproteins from Gelidium pusillum can be achieved by a combination of ultrasound with maceration [276].

The safety of cosmetic products can be ensured by maintaining quality control and standardization. Some macroalgae species have high cosmetic value due to their highly diversified molecules and are required to evaluate the presence and concentration of heavy metals and pesticides. The sterilization level must be high, as set by the WHO and US FDA (Food and Drug Administration) [266]. One of the precautions to be reported in preparing cosmetic formulations is the harmful effect of toxicity which arises due to the presence of phototoxic compounds or photo allergens. These compounds may become activated by coming in contact with the skin and light exposure. Many of these compounds are oxidants and produce ROS [277]. People are not aware that these natural cosmetic products are a combination of many natural raw materials and chemical components that might develop unfavourable effects. Thus, after in vitro and in vivo experiments of prepared formulation, a clinical investigation has to be performed to determine the well-being and effectiveness of the product on human health. It is believed that the cosmeceutical sectors would become significantly enhanced in forthcoming years [278]. Therefore, a deeper level of investigation is required to formulate better cosmetic products.

\section{Conclusions}

Marine macroalgae-derived bioactive constituents have been applicable in cosmeceuticals applications due to their potential skin benefits. This wide variety of applicability and skin benefits enhances interest in and potentiality for use of seaweed-based constituents in cosmetic products. However, it is well demonstrated that cosmetic companies have become more interested in use of marine macroalgae by extracting active compounds and 
their applications in cosmetic preparations. Macroalgae are a valuable resource of bioactive components, with scientific evidence revealing their benefits for safer use in humans and wellbeing. Marine algae-derived molecules showed biological effects on the skin, such as skin whitening, antiaging, antiwrinkle, photoprotection, moisturizing, and collagenboosting, anti-inflammatory, antimicrobial, anticellulite, antiviral, and anticancer activities. Moreover, many cosmeceutical companies already use marine algae extracts and have derived compounds from these extracts in their formulations. However, the biochemical profile monitoring of macroalgae presents a problem that industries need to overcome. The development of its cultivation and sustainable methods of extraction procedures shows the significant key for this confined, which is being analyzed with noteworthy benefits. However, more detail analysis requires to understand the exact mechanism of some compounds since some compounds have not been fully explored. Therefore, the further analysis and evaluation are essential to improve the quality of cosmetic formulations which will be useful to enhance consumers safety.

Author Contributions: Conceptualization, H.S.K. and L.P.; methodology, H.S.K.; investigation, H.S.K. and N.B.P.; writing-original draft preparation, H.S.K. and N.B.P.; writing-review and editing, H.S.K. and L.P. All authors have read and agreed to the published version of the manuscript.

Funding: This research received no external funding.

Acknowledgments: This work had the support of "Fundação para a Ciência e Tecnologia (FCT)", through the strategic projects (No. UID/MAR/04292/2020) granted to MARE.

Conflicts of Interest: The authors declare no conflict of interest.

\section{References}

1. Kligman, D. Cosmeceuticals. Dermatol. Clin. 2000, 18, 609-615. [CrossRef]

2. Dureja, H.; Kaushik, D.; Gupta, M.; Kumar, V.; Lather, V. Cosmeceuticals: An emerging concept. Indian J. Pharmacol. $2005,37,155$. [CrossRef]

3. Kerdudo, A.; Burger, P.; Merck, F.; Dingas, A.; Rolland, Y.; Michel, T.; Fernandez, X. Développement d'un ingrédient naturel: Étude de cas d'un conservateur naturel. Comptes. Rendus. Chim. 2016, 19, 1077-1089. [CrossRef]

4. Barrett, J.R. Chemical exposures: The ugly side of beauty products. Environ. Health Perspect. 2005, 113, 24-27. [CrossRef]

5. Cheong, K.L.; Qiu, H.M.; Du, H.; Liu, Y.; Khan, B.M. Oligosaccharides derived from red seaweed: Production, properties, and potential health and cosmetic applications. Molecules 2018, 23, 2451. [CrossRef]

6. Pereira, J.X.; Pereira, T.C. Cosmetics and its health risks. Glob. J. Med. Res. 2018, 18, 63-70. [CrossRef]

7. Ridder, M. Market Value for Natural and Organic Beauty Worldwide 2018-2027. Available online: https://www.statista.com/ statistics /673641/global-market-value-for-natural-cosmetics / (accessed on 18 November 2020).

8. Mukherjee, P.K.; Maity, N.; Nema, N.K.; Sarkar, B.K. Bioactive compounds from natural resources against skin aging. Phytomedicin 2011, 19, 64-73. [CrossRef]

9. Leelapornpisid, P.; Mungmai, L.; Sirithunyalug, B.; Jiranusornkul, S.; Peerapornpisal, Y. A novel moisturizer extracted from freshwater macroalga [Rhizoclonium hieroglyphicum (C. Agardh) Kützing] for skin care cosmetic. Chiang Mai J. Sci. 2014, 41, 1195-1207.

10. García-Poza, S.; Leandro, A.; Cotas, C.; Cotas, J.; Marques, J.C.; Pereira, L.; Gonçalves, A.M. The evolution road of seaweed aquaculture: Cultivation technologies and the industry 4.0. Int. J. Environ. Res. Public Health 2020, 17, 6528. [CrossRef]

11. Veluchamy, C.H.A.N.D.R.A.; Palaniswamy, R.A.D.H.A. A review on marine algae and its applications. Asian J. Pharm. Clin. Res. 2020, 13, 21-27. [CrossRef]

12. Fu, W.; Nelson, D.R.; Yi, Z.; Xu, M.; Khraiwesh, B.; Jijakli, K.; Chaiboonchoe, A.; Alzahmi, A.; Al-Khairy, D.; Brynjolfsson, S.; et al. Bioactive compounds from microalgae: Current development and prospects. In Studies in Natural Products Chemistry; Elsevier: Amsterdam, The Netherlands, 2017; Volume 54, pp. 199-225.

13. Couteau, C.; Coiffard, L. Phycocosmetics and other marine cosmetics, specific cosmetics formulated using marine resources. Mar. Drugs 2020, 18, 322. [CrossRef]

14. Alhajj, M.J.; Montero, N.; Yarce, C.J.; Salamanca, C.H. Lecithins from vegetable, land, and marine animal sources and their potential applications for cosmetic, food, and pharmaceutical sectors. Cosmetics 2020, 7, 87. [CrossRef]

15. Pallela, R.; Na-Young, Y.; Kim, S.K. Anti-photoaging and photoprotective compounds derived from marine organisms. Mar. Drugs 2010, 8, 1189-1202. [CrossRef] [PubMed]

16. Freitas, R.; Martins, A.; Silva, J.; Alves, C.; Pinteus, S.; Alves, J.; Teodoro, F.; Ribeiro, H.M.; Gonçalves, L.; Petrovski, Ž.; et al. Highlighting the biological potential of the brown seaweed Fucus spiralis for skin applications. Antioxidants 2020, 9, 611. [CrossRef] 
17. Fernando, I.S.; Kim, M.; Son, K.T.; Jeong, Y.; Jeon, Y.J. Antioxidant activity of marine algal polyphenolic compounds: A mechanistic approach. J. Med. Food 2016, 19, 615-628. [CrossRef]

18. Indira, K.; Balakrishnan, S.; Srinivasan, M.; Bragadeeswaran, S.; Balasubramanian, T. Evaluation of in vitro antimicrobial property of seaweed (Halimeda tuna) from Tuticorin coast, Tamil Nadu, Southeast coast of India. Afr. J. Biotechnol. 2013, 12, $284-289$. [CrossRef]

19. Liu, N.; Fu, X.; Duan, D.; Xu, J.; Gao, X.; Zhao, L. Evaluation of bioactivity of phenolic compounds from the brown seaweed of Sargassum fusiforme and development of their stable emulsion. J. Appl. Phycol. 2018, 30, 1955-1970. [CrossRef]

20. Brunt, E.G.; Burgess, J.G. The promise of marine molecules as cosmetic active ingredients. Int. J. Cosmet. Sci. 2018, 40, 1-15. [CrossRef]

21. Percival, E. The polysaccharides of green, red and brown seaweeds: Their basic structure, biosynthesis and function. Br. Phycol. J. 1979, 14, 103-117. [CrossRef]

22. Fernando, I.S.; Sanjeewa, K.A.; Samarakoon, K.W.; Lee, W.W.; Kim, H.S.; Kang, N.; Ranasinghe, P.; Lee, H.S.; Jeon, Y.J. A fucoidan fraction purified from Chnoospora minima; a potential inhibitor of LPS-induced inflammatory responses. Int. J. Biol. Macromol. 2017, 104, 1185-1193. [CrossRef]

23. Ariede, M.B.; Candido, T.M.; Jacome, A.L.M.; Velasco, M.V.R.; de Carvalho, J.C.M.; Baby, A.R. Cosmetic attributes of algae-A review. Algal Res. 2017, 25, 483-487. [CrossRef]

24. Wang, Z.J.; Xu, W.; Liang, J.W.; Wang, C.S.; Kang, Y. Effect of fucoidan on B16 murine melanoma cell melanin formation and apoptosis. Afr. J. Tradit. Complement. Altern. Med. 2017, 14, 149-155. [CrossRef] [PubMed]

25. Teas, J.; Irhimeh, M.R. Melanoma and brown seaweed: An integrative hypothesis. J. Appl. Phycol. 2017, 29, 941-948. [CrossRef] [PubMed]

26. Ghorbanzadeh, B.; Mansouri, M.T.; Hemmati, A.A.; Naghizadeh, B.; Mard, S.A.; Rezaie, A. Mechanism underlying the antiinflammatory effect of sulphated polysaccharide from Padina tetrastromatica against carrageenan induced paw edema in rats. Indian J. Pharmacol. 2015, 47, 292-298. [CrossRef] [PubMed]

27. Khan, M.N.; Yoon, S.J.; Choi, J.S.; Park, N.G.; Lee, H.H.; Cho, J.Y.; Hong, Y.K. Anti-edema effects of brown seaweed (Undaria pinnatifida) extract on phorbol 12-myristate 13-acetate-induced mouse ear inflammation. Am. J. Chin. Med. 2009, 37, 373-381. [CrossRef]

28. Vasconcelos, J.B.; de Vasconcelos, E.R.; Urrea-Victoria, V.; Bezerra, P.S.; Reis, T.N.; Cocentino, A.L.; Navarro, D.M.; Chow, F.; Areces, A.J.; Fujii, M.T. Antioxidant activity of three seaweeds from tropical reefs of Brazil: Potential sources for bioprospecting. $J$. Appl. Phycol. 2019, 31, 835-846. [CrossRef]

29. Santos, J.P.; Torres, P.B.; dos Santos, D.Y.; Motta, L.B.; Chow, F. Seasonal effects on antioxidant and anti-HIV activities of Brazilian seaweeds. J. Appl. Phycol. 2019, 31, 1333-1341. [CrossRef]

30. Kim, J.A.; Ahn, B.N.; Kong, C.S.; Kim, S.K. The chromene sargachromanol E inhibits ultraviolet A-induced ageing of skin in human dermal fibroblasts. Br. J. Dermatol. 2013, 168, 968-976. [CrossRef]

31. Azam, M.S.; Choi, J.; Lee, M.S.; Kim, H.R. Hypopigmenting effects of brown algae-derived phytochemicals: A review on molecular mechanisms. Mar. Drugs 2017, 15, 297. [CrossRef]

32. Yoon, H.S.; Koh, W.B.; Oh, Y.S.; Kim, I.J. The Anti-melanogenic effects of Petalonia binghamiae extracts in $\alpha$-melanocyte stimulating hormone-induced B16/F10 murine melanoma cells. J. Korean Soc. Appl. Biol. Chem. 2009, 52, 564-567. [CrossRef]

33. Wang, H.M.; Li, X.C.; Lee, D.J.; Chang, J.S. Potential biomedical applications of marine algae. Bioresour. Technol. 2017, 244, 1407-1415. [CrossRef] [PubMed]

34. Sakai, S.; Komura, Y.; Nishimura, Y.; Sugawara, T.; Hirata, T. Inhibition of mast cell degranulation by phycoerythrin and its pigment moiety phycoerythrobilin, prepared from Porphyra yezoensis. Food Sci. Technol. Res. 2011, 17, 171-177. [CrossRef]

35. Hartmann, A.; Gostner, J.; Fuchs, J.E.; Chaita, E.; Aligiannis, N.; Skaltsounis, L.; Ganzera, M. Inhibition of collagenase by mycosporine-like amino acids from marine sources. Planta Med. 2015, 81, 813-820. [CrossRef] [PubMed]

36. Li, K.; Li, X.M.; Gloer, J.B.; Wang, B.G. New nitrogen-containing bromophenols from the marine red alga Rhodomela confervoides and their radical scavenging activity. Food Chem. 2012, 135, 868-872. [CrossRef] [PubMed]

37. Wang, H.M.D.; Chen, C.C.; Huynh, P.; Chang, J.S. Exploring the potential of using algae in cosmetics. Bioresour. Technol. 2015, 84, 355-362. [CrossRef] [PubMed]

38. Venkatesan, J.; Anil, S.; Kim, S.K. Introduction to Seaweed Polysaccharides. In Seaweed Polysaccharides-Isolation, Biological and Biomedical Applications, 1st ed.; Venkatesan, J., Anil, S., Kim, S.-K., Eds.; Elsevier: Amsterdam, The Netherlands, 2017. [CrossRef]

39. Robic, A.; Rondeau-Mouro, C.; Sassi, J.F.; Lerat, Y.; Lahaye, M. Structure and interactions of ulvan in the cell wall of the marine green algae Ulva rotundata (Ulvales, Chlorophyceae). Carbohydr. Polym. 2009, 77, 206-216. [CrossRef]

40. Jiang, Z.; Hama, Y.; Yamaguchi, K.; Oda, T. Inhibitory effect of sulphated polysaccharide porphyran on nitric oxide production in lipopolysaccharide-stimulated RAW264. 7 macrophages. J. Biochem. 2012, 151, 65-74. [CrossRef]

41. Isaka, S.; Cho, K.; Nakazono, S.; Abu, R.; Ueno, M.; Kim, D.; Oda, T. Antioxidant and anti-inflammatory activities of porphyran isolated from discolored nori (Porphyra yezoensis). Int. J. Biol. Macromol. 2015, 74, 68-75. [CrossRef]

42. Raposo, M.F.; De Morais, R.M.; de Morais, A.M.B. Bioactivity and applications of sulphated polysaccharides from marine microalgae. Mar. Drugs 2013, 11, 233-252. [CrossRef]

43. Kim, S.-K.; Ravichandran, Y.D.; Khan, S.B.; Kim, Y.T. Prospective of the cosmeceuticals derived from marine organisms. Biotechnol. Bioprocess Eng. 2008, 13, 511-523. [CrossRef] 
44. Pangestuti, R.; Shin, K.H.; Kim, S.K. Anti-photoaging and potential skin health benefits of seaweeds. Mar. Drugs 2021, $19,172$. [CrossRef]

45. Jesumani, V.; Du, H.; Pei, P.; Zheng, C.; Cheong, K.L.; Huang, N. Unravelling property of polysaccharides from Sargassum s as an anti-wrinkle and skin whitening property. Int. J. Biol. Macromol. 2019, 140, 216-224. [CrossRef] [PubMed]

46. Morya, V.K.; Kim, J.; Kim, E.K. Algal fucoidan: Structural and size-dependent bioactivities and their perspectives. Appl. Microbiol. Biotechnol. 2012, 93, 71-82. [CrossRef]

47. Sanjeewa, K.A.; Kang, N.; Ahn, G.; Jee, Y.; Kim, Y.T.; Jeon, Y.J. Bioactive potentials of sulfated polysaccharides isolated from brown seaweed Sargassum spp in related to human health applications: A review. Food Hydrocoll. 2018, 81, 200-208. [CrossRef]

48. Rupérez, P.; Ahrazem, O.; Leal, J.A. Potential antioxidant capacity of sulfated polysaccharides from the edible marine brown seaweed Fucus vesiculosus. J. Agric. Food Chem. 2002, 50, 840-845. [CrossRef] [PubMed]

49. Zhang, Q.; Li, N.; Zhou, G.; Lu, X.; Xu, Z.; Li, Z. In vivo antioxidant activity of polysaccharide fraction from Porphyra haitanesis (Rhodophyta) in aging mice. Pharmacol. Res. 2003, 48, 151-155. [CrossRef]

50. Zhang, Q.; Li, N.; Liu, X.; Zhao, Z.; Li, Z.; Xu, Z. The structure of a sulfated galactan from Porphyra haitanensis and its in vivo antioxidant activity. Carbohydr. Res. 2004, 339, 105-111. [CrossRef] [PubMed]

51. Ayoub, A.; Pereira, J.M.; Rioux, L.E.; Turgeon, S.L.; Beaulieu, M.; Moulin, V.J. Role of seaweed laminaran from Saccharina longicruris on matrix deposition during dermal tissue-engineered production. Int. J. Biol. Macromol. 2015, 75, 13-20. [CrossRef]

52. Ozanne, H.; Toumi, H.; Roubinet, B.; Landemarre, L.; Lespessailles, E.; Daniellou, R.; Cesaro, A. Laminarin effects, a $\beta$-(1,3)-Glucan, on skin cell inflammation and oxidation. Cosmetics 2020, 7, 66. [CrossRef]

53. Rioux, L.E.; Moulin, V.; Beaulieu, M.; Turgeon, S.L. Human skin fibroblast response is differentially regulated by galactofucan and low molecular weight galactofucan. Bioact. Carbohydr. Diet. Fibre 2013, 1, 105-110. [CrossRef]

54. Thevanayagam, H.; Mohamed, S.M.; Chu, W.L. Assessment of UVB-photoprotective and antioxidative activities of carrageenan in keratinocytes. J. Appl. Phycol. 2014, 26, 1813-1821. [CrossRef]

55. Wells, M.L.; Potin, P.; Craigie, J.S.; Raven, J.A.; Merchant, S.S.; Helliwell, K.E.; Smith, A.G.; Camire, M.E.; Brawley, S.H. Algae as nutritional and functional food sources: Revisiting our understanding. J. Appl. Phycol. 2017, 29, 949-982. [CrossRef] [PubMed]

56. Pereira, L.; Gheda, S.F.; Ribeiro-Claro, P.J. Analysis by vibrational spectroscopy of seaweed polysaccharides with potential use in food, pharmaceutical, and cosmetic industries. Int. J. Carbohydr. Chem. 2013, 2013, 537202. [CrossRef]

57. Yang, J.H. Topical application of fucoidan improves atopic dermatitis symptoms in NC/Nga mice. Phytother. Res. 2012, 26, 1898-1903. [CrossRef]

58. Yang, Y.; Liu, D.; Wu, J.; Chen, Y.; Wang, S. In vitro antioxidant activities of sulfated polysaccharide fractions extracted from Corallina officinalis. Int. J. Biol. Macromol. 2011, 49, 1031-1037. [CrossRef]

59. Fournière, M.; Bedoux, G.; Lebonvallet, N.; Leschiera, R.; Le Goff-Pain, C.; Bourgougnon, N.; Latire, T. Poly-and oligosaccharide Ulva s fractions from enzyme-assisted extraction modulate the metabolism of extracellular matrix in human skin fibroblasts: Potential in anti-aging dermo-cosmetic applications. Mar. Drugs 2021, 19, 156. [CrossRef]

60. Jiang, N.; Li, B.; Wang, X.; Xu, X.; Liu, X.; Li, W.; Chang, X.; Li, H.; Qi, H. The antioxidant and antihyperlipidemic activities of phosphorylated polysaccharide from Ulva pertusa. Int. J. Biol. Macromol. 2020, 145, 1059-1065. [CrossRef]

61. Quinderé, A.L.; Fontes, B.P.; de SO Vanderlei, E.; de Queiroz, I.N.; Rodrigues, J.A.; de Araújo, I.W.; Jorge, R.J.; de Menezes, D.B.; e Silva, A.A.; Chaves, H.V.; et al. Peripheral antinociception and anti-edematogenic effect of a sulfated polysaccharide from Acanthophora muscoides. Pharmacol. Rep. 2013, 65, 600-613. [CrossRef]

62. Rodrigues, J.A.G.; de Queiroz, I.N.L.; Quinderé, A.L.G.; Benevides, N.M.B.; Tovar, A.M.F.; de Souza Mourão, P.A. Extraction and structural properties of Acanthophora muscoides (Rhodophyceae) extracellular matrix sulfated polysaccharides and their effects on coagulation. Acta Sci. Technol. 2016, 38, 273-282. [CrossRef]

63. Rodrigues, J.A.; de Queiroz, I.N.; Quinderé, A.L.; Benevides, N.M.; Tovar, A.M.; de Souza Mourão, P.A. Mild-acid hydrolysis of a native polysulfated fraction from Acanthophora muscoides generates sulfated oligosaccharides displaying in vitro thrombin generation inhibition. Acta Sci. Biol. Sci. 2016, 38, 7-15. [CrossRef]

64. Stengel, D.B.; Connan, S. Marine algae: A source of biomass for biotechnological applications. In Natural Products from Marine Algae. Methods in Molecular Biology; Humana Press: New York, NY, USA, 2015; Volume 1308, pp. 1-37. [CrossRef]

65. Lahaye, M.; Robic, A. Structure and functional properties of ulvan, a polysaccharide from green seaweeds. Biomacromolecules 2007, 8, 1765-1774. [CrossRef] [PubMed]

66. Wijesinghe, W.A.; Jeon, Y.J. Biological activities and potential industrial applications of fucose rich sulfated polysaccharides and fucoidans isolated from brown seaweeds: A review. Carbohydr. Polym. 2012, 88, 13-20. [CrossRef]

67. Dita, L.R.; Triastuti, J. Utilization of agar Gracilaria sp. as a natural thickener on liquid bath soap formulation. IOP Conf. Ser. Earth Environ. Sci. 2020, 441, 012021. [CrossRef]

68. Kordjazi, M.; Shabanpour, B.; Zabihi, E.; Faramarzi, M.A.; Feizi, F.; Gavlighi, H.A.; Feghhi, M.A.; Hosseini, S.A. Sulfated polysaccharides purified from two species of Padina improve collagen and epidermis formation in the rat. Int. J. Mol. Cell. Med. 2013, 2, 156-163.

69. Malinowska, P. Algae extracts as active cosmetic ingredients. Zesz. Nauk. Uniw. Ekon. Pozn. 2011, 212, 123-129.

70. Tønnesen, H.H.; Karlsen, J. Alginate in drug delivery systems. Drug Dev. Ind. Pharm. 2002, 28, 621-630. [CrossRef]

71. Mizutani, S.; Deguchi, S.; Kobayashi, E.; Nishiyama, E.; Sagawa, H.; Kato, I. Fucoidan-Containing Cosmetics. U.S. Patent 2006/0093566A1, 4 May 2006. 
72. Morais, T.; Cotas, J.; Pacheco, D.; Pereira, L. Seaweeds Compounds: An ecosustainable source of cosmetic ingredients? Cosmetics 2021, 8, 8. [CrossRef]

73. Jesumani, V.; Du, H.; Pei, P.; Aslam, M.; Huang, N. Comparative study on skin protection activity of polyphenol-rich extract and polysaccharide-rich extract from Sargassum vachellianum. PLoS ONE 2020, 15, e0227308. [CrossRef]

74. Rupérez, P. Mineral content of edible marine seaweeds. Food Chem. 2002, 79, 23-26. [CrossRef]

75. Je, J.Y.; Park, P.J.; Kim, E.K.; Park, J.S.; Yoon, H.D.; Kim, K.R.; Ahn, C.B. Antioxidant activity of enzymatic extracts from the brown seaweed Undaria pinnatifida by electron spin resonance spectroscopy. LWT Food Sci. Technol. 2009, 42, 874-878. [CrossRef]

76. Pereira, L. Seaweeds as source of bioactive substances and skin care therapy-Cosmeceuticals, algotheraphy, and thalassotherapy. Cosmetics 2018, 5, 68. [CrossRef]

77. Heo, S.J.; Ko, S.C.; Cha, S.H.; Kang, D.H.; Park, H.S.; Choi, Y.U.; Kim, D.; Jung, W.K.; Jeon, Y.J. Effect of phlorotannins isolated from Ecklonia cava on melanogenesis and their protective effect against photo-oxidative stress induced by UV-B radiation. Toxicol. Vitr. 2009, 23, 1123-1130. [CrossRef] [PubMed]

78. Ko, S.C.; Cha, S.H.; Heo, S.J.; Lee, S.H.; Kang, S.M.; Jeon, Y.J. Protective effect of Ecklonia cava on UVB-induced oxidative stress: In vitro and in vivo zebrafish model. J. Appl. Phycol. 2011, 23, 697-708. [CrossRef]

79. Fernando, I.P.S.; Kim, K.N.; Kim, D.; Jeon, Y.J. Algal polysaccharides: Potential bioactive substances for cosmeceutical applications. Crit. Rev. Biotechnol. 2019, 39, 99-113. [CrossRef] [PubMed]

80. Xue, C.; Yu, G.; Hirata, T.; Terao, J.; Lin, H. Antioxidative activities of several marine polysaccharides evaluated in a phosphatidylcholine-liposomal suspension and organic solvents. Biosci. Biotechnol. Biochem. 1998, 62, 206-209. [CrossRef]

81. Berthon, J.Y.; Nachat-Kappes, R.; Bey, M.; Cadoret, J.P.; Renimel, I.; Filaire, E. Marine algae as attractive source to skin care. Free Radic. Res. 2017, 51, 555-567. [CrossRef]

82. Yu, P.; Sun, H. Purification of a fucoidan from kelp polysaccharide and its inhibitory kinetics for tyrosinase. Carbohydr. Polym. 2014, 99, 278-283. [CrossRef]

83. Fernando, I.P.S.; Sanjeewa, K.K.A.; Samarakoon, K.W.; Kim, H.-S.; Gunasekara, U.K.D.S.S.; Park, Y.-J.; Abeytunga, D.T.U.; Lee, W.W.; Jeon, Y.-J. The potential of fucoidans from Chnoospora minima and Sargassum polycystum in cosmetics: Antioxidant, anti-inflammatory, skin-whitening, and antiwrinkle activities. J. Appl. Phycol. 2018, 30, 3223-3232. [CrossRef]

84. Park, E.J.; Choi, J.I. Melanogenesis inhibitory effect of low molecular weight fucoidan from Undaria pinnatifida. J. Appl. Phycol. 2017, 29, 2213-2217. [CrossRef]

85. Wang, L.; Lee, W.; Oh, J.; Cui, Y.; Ryu, B.; Jeon, Y.J. Protective effect of sulfated polysaccharides from celluclast-assisted extract of Hizikia fusiforme against ultraviolet B-induced skin damage by regulating NF- $\mathrm{B}$, AP-1, and MAPKs signalling pathways in vitro in human dermal fibroblasts. Mar. Drugs 2018, 16, 239. [CrossRef]

86. Moon, H.J.; Lee, S.R.; Shim, S.N.; Jeong, S.H.; Stonik, V.A.; Rasskazov, V.A.; Zvyagintseva, T.; Lee, Y.H. Fucoidan inhibits UVB-induced MMP-1 expression in human skin fibroblasts. Biol. Pharm. Bull. 2008, 31, 284-289. [CrossRef] [PubMed]

87. Moon, H.J.; Lee, S.H.; Ku, M.J.; Yu, B.C.; Jeon, M.J.; Jeong, S.H.; Stonik, V.A.; Zvyagintseva, T.; Ermakova, S.P.; Lee, Y.H. Fucoidan inhibits UVB-induced MMP-1 promoter expression and down regulation of type I procollagen synthesis in human skin fibroblasts. Eur. J. Dermatol. 2009, 19, 129-134. [CrossRef]

88. Jesumani, V.; Du, H.; Aslam, M.; Pei, P.; Huang, N. Potential use of seaweed bioactive compounds in skincare-A review. Mar. Drugs 2019, 17, 688. [CrossRef] [PubMed]

89. Shao, P.; Shao, J.; Han, L.; Lv, R.; Sun, P. Separation, preliminary characterization, and moisture-preserving activity of polysaccharides from Ulva fasciata. Int. J. Biol. Macromol. 2015, 72, 924-930. [CrossRef]

90. Wang, J.; Jin, W.; Hou, Y.; Niu, X.; Zhang, H.; Zhang, Q. Chemical composition and moisture-absorption/retention ability of polysaccharides extracted from five algae. Int. J. Biol. Macromol. 2013, 57, 26-29. [CrossRef] [PubMed]

91. Ruxton, C.H.; Jenkins, G. A novel topical ingredient derived from seaweed significantly reduces symptoms of Acne vulgaris: A general literature review. J. Cosmet. Sci. 2013, 64, 219-226.

92. Sebaaly, C.; Kassem, S.; Grishina, E.; Kanaan, H.; Sweidan, A.; Chmit, M.S.; Kanaan, H.M. Anticoagulant and antibacterial activities of polysaccharides of red algae Corallina collected from Lebanese coast. J. Appl. Pharm. Sci. 2014, 4, 30. [CrossRef]

93. Admassu, H.; Gasmalla, M.A.A.; Yang, R.; Zhao, W. Bioactive peptides derived from seaweed protein and their health benefits: Antihypertensive, antioxidant, and antidiabetic properties. J. Food Sci. 2018, 83, 6-16. [CrossRef]

94. Stengel, D.B.; Connan, S.; Popper, Z.A. Algal chemodiversity and bioactivity: Sources of natural variability and implications for commercial application. Biotechnol. Adv. 2011, 29, 483-501. [CrossRef]

95. Houston, M.C. Nutraceuticals, vitamins, antioxidants, and minerals in the prevention and treatment of hypertension. Prog. Cardiovasc. Dis. 2005, 47, 396-449. [CrossRef]

96. Bedoux, G.; Hardouin, K.; Burlot, A.S.; Bourgougnon, N. Bioactive components from seaweeds: Cosmetic applications and future development. Adv. Bot. Res. 2014, 71, 345-378. [CrossRef]

97. Heo, S.J.; Lee, K.W.; Song, C.B.; Jean, Y.J. Antioxidant activity of enzymatic extracts from brown seaweeds. Bioresour. Technol. 2005, 96, 1613-1623. [CrossRef] [PubMed]

98. Gupta, P.L.; Rajput, M.; Oza, T.; Trivedi, U.; Sanghvi, G. Eminence of microbial products in cosmetic industry. Nat. Prod. Bioprospect. 2019, 9, 267-278. [CrossRef] [PubMed] 
99. Salehi, B.; Sharifi-Rad, J.; Seca, A.M.L.; Pinto, D.C.G.A.; Michalak, I.; Trincone, A.; Mishra, A.P.; Nigam, M.; Zam, W.; Martins, N. Current trends on seaweeds: Looking at chemical composition, phytopharmacology, and cosmetic applications. Molecules 2019, 24, 4182. [CrossRef]

100. Barceló-Villalobos, M.; Figueroa, F.L.; Korbee, N.; Álvarez-Gómez, F.; Abreu, M.H. Production of mycosporine-like amino acids from Gracilaria vermiculophylla (Rhodophyta) cultured through one year in an integrated multi-trophic aquaculture (IMTA) system. Mar. Biotechnol. 2017, 19, 246-254. [CrossRef]

101. Leandro, A.; Pereira, L.; Gonçalves, A.M. Diverse applications of marine macroalgae. Mar. Drugs 2020, 18, 17. [CrossRef]

102. Hupel, M.; Lecointre, C.; Meudec, A.; Poupart, N.; Gall, E.A. Comparison of photoprotective responses to UV radiation in the brown seaweed Pelvetia canaliculata and the marine angiosperm Salicornia ramosissima. J. Exp. Mar. Biol. Ecol. 2011, $401,36-47$. [CrossRef]

103. Orfanoudaki, M.; Hartmann, A.; Karsten, U.; Ganzera, M. Chemical profiling of mycosporine-like amino acids in twenty-three red algal species. J. Phycol. 2019, 55, 393-403. [CrossRef]

104. Guglielmo, M.; Montanari, D. Cosmetic Composition with a Lifting Effect for Sustaining Relaxed Tissues. Patent WO2008146116 A2, 4 December 2008. Available online: https:/ / patents.google.com/patent/WO2008146116A3/tr (accessed on 27 December 2021).

105. Daniel, S.; Cornelia, S.; Fred, Z. UV-A sunscreen from red algae for protection against premature skin aging. Cosmet. Toilet. Manuf. Worldw. 2004, 2004, 139-143.

106. Hartmann, A.; Becker, K.; Karsten, U.; Remias, D.; Ganzera, M. Analysis of mycosporine-like amino acids in selected algae and cyanobacteria by hydrophilic interaction liquid chromatography and a novel MAA from the red alga Catenella repens. Mar. Drugs 2015, 13, 6291-6305. [CrossRef]

107. Gao, Q.; Garcia-Pichel, F. Microbial ultraviolet sunscreens. Nat. Rev. Microbiol. 2011, 9, 791-802. [CrossRef]

108. Rangel, K.C.; Villela, L.Z.; de Castro Pereira, K.; Colepicolo, P.; Debonsi, H.M.; Gaspar, L.R. Assessment of the photoprotective potential and toxicity of Antarctic red macroalgae extracts from Curdiea racovitzae and Iridaea cordata for cosmetic use. Algal Res. 2020, 50, 101984. [CrossRef]

109. Hagino, H.; Saito, M. Use of Algal Proteins in Cosmetics. European Patent EP1433463B1, 22 September 2010. Available online: https:/ / patents.google.com/patent/EP1433463B1/en (accessed on 27 December 2021).

110. Fleurence, J. Seaweed proteins. In Proteins in Food Processing, 1st ed.; Yada, R.Y., Ed.; Woodhead Publishing: Cambridge, UK, 2004; pp. 197-213.

111. Athukorala, Y.; Trang, S.; Kwok, C.; Yuan, Y.V. Antiproliferative and antioxidant activities and mycosporine-like amino acid profiles of wild-harvested and cultivated edible Canadian marine red macroalgae. Molecules 2016, 21, E119. [CrossRef] [PubMed]

112. Pereira, L. Characterization of Bioactive Components in Edible Algae, 1st ed.; Marine Drugs MDPI: Basel, Switzerland, 2020. [CrossRef]

113. Gedouin, A.; Valle, R.; Morvan, P.Y. Use of Algae Extract to Stimulate the Oxygen Uptake by the Cells Having Lipolytic Effect to Produce ATP Molecules. Patent FR2879098 A1, 16 June 2006. Available online: https://www.lens.org/lens/patent/FR_2879098_ A1 (accessed on 28 October 2021).

114. Lee, H.A.; Kim, I.H.; Nam, T.J. Bioactive peptide from Pyropia yezoensis and its anti-inflammatory activities Int. J. Mol. Med. 2015, 36, 1701-1706. [CrossRef]

115. Yuan, Y.V.; Westcott, N.D.; Hu, C.; Kitts, D.D. Mycosporine-like amino acid composition of the edible red alga, Palmaria palmata (dulse) harvested from the west and east coasts of Grand Manan Island, New Brunswick. Food Chem. 2009, 112, 321-328. [CrossRef]

116. Song, T.Y.; Chen, C.H.; Yang, N.C.; Fu, C.S. The correlation of in vitro mushroom tyrosinase activity with cellular tyrosinase activity and melanin formation in melanoma cells A2058. J. Food Drug Anal. 2009, 17, 4. [CrossRef]

117. Chan, Y.Y.; Kim, K.H.; Cheah, S.H. Inhibitory effects of Sargassum polycystum on tyrosinase activity and melanin formation in B16F10 murine melanoma cells. J. Ethnopharmacol. 2011, 137, 1183-1188. [CrossRef] [PubMed]

118. Gianeti, M.D.; Maia Campos, P.M. Efficacy evaluation of a multifunctional cosmetic formulation: The benefits of a combination of active antioxidant substances. Molecules 2014, 19, 18268-18282. [CrossRef]

119. Ryu, J.; Park, S.J.; Kim, I.H.; Choi, Y.H.; Nam, T.J. Protective effect of porphyra-334 on UVA-induced photoaging in human skin fibroblasts. Int. J. Mol. Med. 2014, 34, 796-803. [CrossRef]

120. Vega, J.; Schneider, G.; Moreira, B.R.; Herrera, C.; Bonomi-Barufi, J.; Figueroa, F.L. Mycosporine-like amino acids from red macroalgae: UV-photoprotectors with potential cosmeceutical applications. Appl. Sci. 2021, 11, 5112. [CrossRef]

121. Nishida, Y.; Kumagai, Y.; Michiba, S.; Yasui, H.; Kishimura, H. Efficient extraction and antioxidant capacity of mycosporine-like amino acids from red alga Dulse Palmaria palmata in Japan. Mar. Drugs 2020, 18, 502. [CrossRef]

122. Pangestuti, R.; Siahaan, E.A.; Kim, S.K. Photoprotective substances derived from marine algae. Mar. Drugs 2018, 16, 399. [CrossRef] [PubMed]

123. Kim, C.R.; Kim, Y.M.; Lee, M.K.; Kim, I.H.; Choi, Y.H.; Nam, T.J. Pyropia yezoensis peptide promotes collagen synthesis by activating the TGF- $\beta$ /Smad signaling pathway in the human dermal fibroblast cell line Hs27. Int. J. Mol. Med. 2017, 39, 31-38. [CrossRef] [PubMed]

124. Cardozo, K.H.; Marques, L.G.; Carvalho, V.M.; Carignan, M.O.; Pinto, E.; Marinho-Soriano, E.; Colepicolo, P. Analyses of photoprotective compounds in red algae from the Brazilian coast. Rev. Bras. Farmacogn. 2011, 21, 202-208. [CrossRef] 
125. Hoyer, K.; Karsten, U.; Sawall, T.; Wiencke, C. Photoprotective substances in Antarctic macroalgae and their variation with respect to depth distribution, different tissues and developmental stages. Mar. Ecol. Prog. Ser. 2001, 211, 117-129. [CrossRef]

126. Oren, A.; Gunde-Cimerman, N. Mycosporines and mycosporine-like amino acids: UV protectants or multipurpose secondary metabolites? FEMS Microbiol. Lett. 2007, 269, 1-10. [CrossRef]

127. Mercurio, D.G.; Wagemaker, T.A.L.; Alves, V.M.; Benevenuto, C.G.; Gaspar, L.R.; Campos, P.M. In vivo photoprotective effects of cosmetic formulations containing UV filters, vitamins, Ginkgo biloba and red algae extracts. J. Photochem. Photobiol. B Biol. 2015, 153, 121-126. [CrossRef]

128. Suh, S.S.; Hwang, J.; Park, M.; Seo, H.H.; Kim, H.S.; Lee, J.H.; Moh, S.H.; Lee, T.K. Anti-inflammation activities of mycosporine-like amino acids (MAAs) in response to UV radiation suggest potential anti-skin aging activity. Mar. Drugs 2014, 12, 5174-5187. [CrossRef]

129. de Andrade, C.J.; de Andrade, L.M. An overview on the application of genus Chlorella in biotechnological processes. J. Adv. Res. Biotechnol. 2017, 2, 1-9. [CrossRef]

130. Kang, K.A.; Lee, K.H.; Chae, S.; Koh, Y.S.; Yoo, B.S.; Kim, J.H.; Ham, Y.M.; Baik, J.S.; Lee, N.H.; Hyun, J.W. Triphlorethol-A from Ecklonia cava protects V79-4 lung fibroblast against hydrogen peroxide induced cell damage. Free Radic. Res. 2005, 39, 883-892. [CrossRef] [PubMed]

131. de Jesus Raposo, M.F.; De Morais, A.M.; De Morais, R.M. Marine polysaccharides from algae with potential biomedical applications. Mar. Drugs 2015, 13, 2967-3028. [CrossRef] [PubMed]

132. Yan, X.; Chuda, Y.; Suzuki, M.; Nagata, T. Fucoxanthin as the major antioxidant in Hijikia fusiformis, a common edible seaweed. Biosci. Biotechnol. Biochem. 1999, 63, 605-607. [CrossRef] [PubMed]

133. Couteau, C.; Coiffard, L. Seaweed application in cosmetics. In Seaweed in Health and Disease Prevention, 1st ed.; Fleurence, J., Levine, I., Eds.; Academic Press: San Diego, CA, USA, 2016; pp. 423-441. [CrossRef]

134. Hoi, Y.H.; Nam, T.-J.; Subramaniyan, S.A.; Begum, N.; Kim, S.J. Biopeptides of Pyropia yezoensis and their potential health benefits: A review. Asian Pac. J. Trop. Biomed. 2021, 11, 375. [CrossRef]

135. Kim, M.S.; Oh, G.H.; Kim, M.J.; Hwang, J.K. Fucosterol inhibits matrix metalloproteinase expression and promotes type-1 procollagen production in UVB-induced HaCaT cells. Photochem. Photobiol. 2013, 89, 911-918. [CrossRef] [PubMed]

136. Hama, S.; Takahashi, K.; Inai, Y.; Shiota, K.; Sakamoto, R.; Yamada, A.; Tsuchiya, H.; Kanamura, K.; Yamashita, E.; Kogure, K. Protective effects of topical application of a poorly soluble antioxidant astaxanthin liposomal formulation on ultraviolet-induced skin damage. J. Pharm. Sci. 2012, 101, 2909-2916. [CrossRef]

137. Pereira, L. Guia Ilustrado das Macroalgas—Conhecer e Reconhecer Algumas Espécies da Flora Portuguesa, 1st ed.; University de Coimbra Press: Coimbra, Portugal, 2009. [CrossRef]

138. Pereira, L. Seaweed flora of the european north atlantic and mediterranean. In Handbook of Marine Biotechnology, 1st ed.; Kim, S.K., Ed.; Springer: Berlin/Heidelberg, Germany, 2015; pp. 65-178. [CrossRef]

139. Christaki, E.; Bonos, E.; Giannenas, I.; Florou-Paneri, P. Functional properties of carotenoids originating from algae. J. Sci. Food Agric. 2013, 15, 5-11. [CrossRef]

140. Carvalho, L.G.; Pereira, L. Review of marine algae as source of bioactive metabolites: A marine biotechnology approach. In Marine Algae-Biodiversity, Taxonomy, Environmental Assessment, and Biotechnology, 1st ed.; Pereira, L., Neto, J.M., Eds.; CRC Press: Boca Raton, FL, USA, 2014; pp. 195-227. [CrossRef]

141. Spolaore, P.; Joannis-Cassan, C.; Duran, E.; Isambert, A. Commercial applications of microalgae. J. Biosci. Bioeng. 2006, 101, 87-96. [CrossRef]

142. Peng, J.; Yuan, J.P.; Wu, C.F.; Wang, J.H. Fucoxanthin, a marine carotenoid present in brown seaweeds and diatoms: Metabolism and bioactivities relevant to human health. Mar. Drugs 2011, 9, 1806-1828. [CrossRef]

143. D'Orazio, N.; Gemello, E.; Gammone, M.A.; De Girolamo, M.; Ficoneri, C.; Riccioni, G. Fucoxantin: A treasure from the sea. Mar. Drugs 2012, 10, 604-616. [CrossRef]

144. Mularczyk, M.; Michalak, I.; Marycz, K. Astaxanthin and other nutrients from Haematococcus pluvialis-Multifunctional applications. Mar. Drugs 2020, 18, 459. [CrossRef]

145. Cardoso, M.S.; Carvalho, L.G.; Silva, P.J.; Rodrigues, M.S.; Pereira, O.R.; Pereira, L. Bioproducts from seaweeds: A review with special focus on the Iberian Peninsula. Curr. Org. Chem. 2014, 18, 896-917. [CrossRef]

146. Kim, D.H.; Eom, S.H.; Kim, T.H.; Kim, B.Y.; Kim, Y.M.; Kim, S.B. Deodorizing effects of phlorotannins from edible brown alga Eisenia bicyclis on methyl mercaptan. J. Agric. Sci. 2013, 5, 95. [CrossRef]

147. Sekar, S.; Chandramohan, M. Phycobiliproteins as a commodity: Trends in applied research, patents and commercialization. J. Appl. Phycol. 2008, 20, 113-136. [CrossRef]

148. Kalasariya, H.S.; Yadav, V.K.; Yadav, K.K.; Tirth, V.; Algahtani, A.; Islam, S.; Gupta, N.; Jeon, B.-H. Seaweed-based molecules and their potential biological activities: An eco-sustainable cosmetics. Molecules 2021, 26, 5313. [CrossRef]

149. Shimoda, H.; Tanaka, J.; Shan, S.J.; Maoka, T. Anti-pigmentary activity of fucoxanthin and its influence on skin mRNA expression of melanogenic molecules. J. Pharm. Pharmacol. 2010, 62, 1137-1145. [CrossRef]

150. Mise, T.; Ueda, M.; Yasumoto, T. Production of fucoxanthin-rich powder from Cladosiphon okamuranus. Adv. J. Food Sci. Technol. 2011, 3, 73-76.

151. Francavilla, M.; Franchi, M.; Monteleone, M.; Caroppo, C. The red seaweed Gracilaria gracilis as a multi products source. Mar. Drugs 2013, 11, 3754-3776. [CrossRef] 
152. Goldberg, S.L. The use of water soluble chlorophyll in oral sepsis: An experimental study of 300 cases. Am. J. Surg. 1943, 62, 117-123. [CrossRef]

153. Spears, K. Developments in food colourings: The natural alternatives. Trends Biotechnol. 1988, 6, 283-288. [CrossRef]

154. Lanfer-Marquez, U.M.; Barros, R.M.; Sinnecker, P. Antioxidant activity of chlorophylls and their derivatives. Food Res. Int. 2005, 38, 885-891. [CrossRef]

155. Le Lann, K.; Surget, G.; Couteau, C.; Coiffard, L.; Cérantola, S.; Gaillard, F.; Larnicol, M.; Zubia, M.; Guérard, F.; Poupart, N.; et al. Sunscreen, antioxidant, and bactericide capacities of phlorotannins from the brown macroalga Halidrys siliquosa. J. Appl. Phycol. 2016, 28, 3547-3559. [CrossRef]

156. Ishihara, K.; Oyamada, C.; Matsushima, R.; Murata, M.; Muraoka, T. Inhibitory effect of porphyran, prepared from dried "Nori”, on contact hypersensitivity in mice. Biosci. Biotechnol. Biochem. 2005, 69, 1824-1830. [CrossRef] [PubMed]

157. López-Hortas, L.; Flórez-Fernández, N.; Torres, M.D.; Ferreira-Anta, T.; Casas, M.P.; Balboa, E.M.; Falqué, E.; Domínguez, H. Applying seaweed compounds in cosmetics, cosmeceuticals and nutricosmetics. Mar. Drugs 2021, 19, 552. [CrossRef] [PubMed]

158. Marquardt, J.; Hanelt, D. Carotenoid composition of Delesseria lancifolia and other marine red algae from polar and temperate habitats. Eur. J. Phycol. 2004, 39, 285-292. [CrossRef]

159. Matsui, M.; Tanaka, K.; Higashiguchi, N.; Okawa, H.; Yamada, Y.; Tanaka, K.; Taira, S.; Aoyama, T.; Takanishi, M.; Natsume, C.; et al. Protective and therapeutic effects of fucoxanthin against sunburn caused by UV irradiation. J. Pharmacol. Sci. 2016, 132, 55-64. [CrossRef]

160. Schubert, N.; García-Mendoza, E.; Pacheco-Ruiz, I. Carotenoid composition of marine red algae. J. Phycol. 2006, 42, 1208-1216. [CrossRef]

161. Heo, S.J.; Jeon, Y.J. Protective effect of fucoxanthin isolated from Sargassum siliquastrum on UV-B induced cell damage. J. Photochem. Photobiol. B 2009, 95, 101-107. [CrossRef]

162. Jiang, H.; Gong, J.; Lou, W.; Dinghui, Z. Photosynthetic behaviors in response to intertidal zone and algal mat density in Ulva lactuca (Chlorophyta) along the coast of Nan'ao Island, Shantou, China. Environ. Sci. Pollut. Res. 2019, 26, 13346-13353. [CrossRef]

163. Rajauria, G.; Foley, B.; Abu-Ghannam, N. Characterization of dietary fucoxanthin from Himanthalia elongata brown seaweed. Food Res. Int. 2017, 99, 995-1001. [CrossRef]

164. Joshi, S.; Kumari, R.; Upasani, V.N. Applications of algae in cosmetics: An overview. Int. J. Innov. Res. Sci. Eng. Technol. 2018, 7,1269 .

165. Zaragozá, M.C.; López, D.P.; Sáiz, M.; Poquet, M.; Pérez, J.; Puig-Parellada, P.; Marmol, F.; Simonetti, P.; Gardana, C.; Lerat, Y.; et al. Toxicity and antioxidant activity in vitro and in vivo of two Fucus vesiculosus extracts. J. Agric. Food Chem. 2008, 56, 7773-7780. [CrossRef] [PubMed]

166. Urikura, I.; Sugawara, T.; Hirata, T. Protective effect of fucoxanthin against UVB-induced skin photoaging in hairless mice. Biosci. Biotechnol. Biochem. 2011, 75, 757-760. [CrossRef]

167. Dunaway, S.; Odin, R.; Zhou, L.; Ji, L.; Zhang, Y.; Kadekaro, A.L. Natural antioxidants: Multiple mechanisms to protect skin from solar radiation. Front. Pharmacol. 2018, 9, 392. [CrossRef] [PubMed]

168. Panayotova, V.; Merzdhanova, A.; Dobreva, D.A.; Zlatanov, M.; Makedonski, L. Lipids of Black Sea algae: Unveiling their potential for pharmaceutical and cosmetic applications. J. IMAB Annu. Proceeding Sci. Pap. 2017, 23, 1747-1751. [CrossRef]

169. Cotas, J.; Leandro, A.; Monteiro, P.; Pacheco, D.; Figueirinha, A.; Gonçalves, A.M.; da Silva, G.J.; Pereira, L. Seaweed phenolics: From extraction to applications. Mar. Drugs 2020, 18, 384. [CrossRef] [PubMed]

170. Gómez-Guzmán, M.; Rodríguez-Nogales, A.; Algieri, F.; Gálvez, J. Potential role of seaweed polyphenols in cardiovascularassociated disorders. Mar. Drugs 2018, 16, 250. [CrossRef]

171. Morais, T.; Inácio, A.; Coutinho, T.; Ministro, M.; Cotas, J.; Pereira, L.; Bahcevandziev, K. Seaweed potential in the animal feed: A review. J. Mar. Sci. Eng. 2020, 8, 559. [CrossRef]

172. Mateos, R.; Pérez-Correa, J.R.; Domínguez, H. Bioactive properties of marine phenolics. Mar. Drugs 2020, 18, 501. [CrossRef]

173. Yang, K.; Kim, S.; Park, J.H.; Ahn, W.G.; Jung, S.H.; Oh, D.; Park, H.C.; Choi, C. Topical application of phlorotannins from brown seaweed mitigates radiation dermatitis in a mouse model. Mar. Drugs 2020, 18, 377. [CrossRef]

174. Thomas, N.V.; Kim, S.K. Potential pharmacological applications of polyphenolic derivatives from marine brown algae. Environ. Toxicol. Pharmacol. 2011, 32, 325-335. [CrossRef]

175. Liu, M.; Hansen, P.E.; Lin, X. Bromophenols in marine algae and their bioactivities. Mar. Drugs 2011, 9, 1273-1292. [CrossRef]

176. Sanjeewa, K.K.; Kim, E.A.; Son, K.T.; Jeon, Y.J. Bioactive properties and potentials cosmeceutical applications of phlorotannins isolated from brown seaweeds: A review. J. Photochem. Photobiol. B Biol. 2016, 162, 100-105. [CrossRef] [PubMed]

177. Fitton, J.H.; Dell'Acqua, G.; Gardiner, V.-A.; Karpiniec, S.S.; Stringer, D.N.; Davis, E. Topical Benefits of Two Fucoidan-Rich Extracts from Marine Macroalgae. Cosmetics 2015, 2, 66-81. [CrossRef]

178. Jang, M.S.; Park, H.Y.; Nam, K.H. Whitening effects of 4-hydroxyphenethyl alcohol isolated from water boiled with Hizikia fusiformis. Food Sci. Biotechnol. 2014, 23, 555-560. [CrossRef]

179. Li, Y.X.; Wijesekara, I.; Li, Y.; Kim, S.K. Phlorotannins as bioactive agents from brown algae. Process. Biochem. 2011, 46, $2219-2224$. [CrossRef]

180. Zou, Y.; Qian, Z.J.; Li, Y.; Kim, M.M.; Lee, S.H.; Kim, S.K. Antioxidant effects of phlorotannins isolated from Ishige okamurae in free radical mediated oxidative systems. J. Agric. Food Chem. 2008, 56, 7001-7009. [CrossRef] [PubMed] 
181. Lee, M.S.; Yoon, H.D.; Kim, J.I.; Choi, J.S.; Byun, D.S.; Kim, H.R. Dioxinodehydroeckol inhibits melanin synthesis through $\mathrm{PI} 3 \mathrm{~K} /$ Akt signalling pathway in $\alpha$-melanocyte-stimulating hormone-treated B16F10 cells. Exp. Dermatol. 2012, 21, 471-473. [CrossRef] [PubMed]

182. Joe, M.J.; Kim, S.N.; Choi, H.Y.; Shin, W.S.; Park, G.M.; Kang, D.W.; Kim, Y.K. The inhibitory effects of eckol and dieckol from Ecklonia stolonifera on the expression of matrix metalloproteinase-1 in human dermal fibroblasts. Biol. Pharm. Bull. 2006, 29, 1735-1739. [CrossRef] [PubMed]

183. Jun, Y.J.; Lee, M.; Shin, T.; Yoon, N.; Kim, J.H.; Kim, H.R. Eckol enhances heme oxygenase-1 expression through activation of Nrf2/JNK pathway in HepG2 cells. Molecules 2014, 19, 15638-15652. [CrossRef]

184. Wijesekara, I.; Yoon, N.Y.; Kim, S.K. Phlorotannins from Ecklonia cava (Phaeophyceae): Biological activities and potential health benefits. Biofactors 2010, 36, 408-414. [CrossRef]

185. Saraf, S.; Kaur, C.D. Phytoconstituents as photoprotective novel cosmetic formulations. Pharmacogn. Rev. 2010, 4, 1-11. [CrossRef] [PubMed]

186. Ryu, B.; Qian, Z.J.; Kim, M.M.; Nam, K.W.; Kim, S.K. Anti-photoaging activity and inhibition of matrix metalloproteinase (MMP) by marine red alga, Corallina pilulifera methanol extract. Radiat. Phys. Chem. 2009, 78, 98-105. [CrossRef]

187. Kong, C.S.; Kim, J.A.; Ahn, B.N.; Kim, S.K. Potential effect of phloroglucinol derivatives from Ecklonia cava on matrix metalloproteinase expression and the inflammatory profile in lipopolysaccharide-stimulated human THP-1 macrophages. Fish Sci. 2011, 77, 867-873. [CrossRef]

188. Leyton, A.; Pezoa-Conte, R.; Barriga, A.; Buschmann, A.H.; Mäki-Arvela, P.; Mikkola, J.P.; Lienqueo, M.E. Identification and efficient extraction method of phlorotannins from the brown seaweed Macrocystis pyrifera using an orthogonal experimental design. Algal Res. 2016, 16, 201-208. [CrossRef]

189. Abu, R.; Jiang, Z.; Ueno, M.; Isaka, S.; Nakazono, S.; Okimura, T.; Cho, K.; Yamaguchi, K.; Kim, D.; Oda, T. Anti-metastatic effects of the sulfated polysaccharide ascophyllan isolated from Ascophyllum nodosum on B16 melanoma. Biochem. Biophys. Res. Commun. 2015, 458, 727-732. [CrossRef]

190. Messina, C.M.; Renda, G.; Laudicella, V.A.; Trepos, R.; Fauchon, M.; Hellio, C.; Santulli, A. From ecology to biotechnology, study of the defense strategies of algae and halophytes (from Trapani Saltworks, NW Sicily) with a focus on antioxidants and antimicrobial properties. Int. J. Mol. Sci. 2019, 20, 881. [CrossRef]

191. Airanthi, M.W.; Hosokawa, M.; Miyashita, K. Comparative antioxidant activity of edible Japanese brown seaweeds. J. Food Sci. 2011, 76, C104-C111. [CrossRef] [PubMed]

192. Vo, T.S.; Kim, S.-K.; Ryu, B.; Ngo, D.; Yoon, N.-Y.; Bach, L.G.; Hang, N.T.N.; Vo, T.S.; Kim, S.-K.; Ryu, B.; et al. The suppressive activity of fucofuroeckol-A derived from brown algal Ecklonia stolonifera Okamura on UVB-induced mast cell degranulation. Mar. Drugs 2018, 16, 1. [CrossRef]

193. Thomas, N.V.; Kim, S.K. Beneficial effects of marine algal compounds in cosmeceuticals. Mar. Drugs 2013, 11, 146-164. [CrossRef]

194. Kim, K.N.; Yang, H.M.; Kang, S.M.; Kim, D.; Ahn, G.; Jeon, Y.J. Octaphlorethol A isolated from Ishige foliacea inhibits $\alpha$-MSHstimulated induced melanogenesis via ERK pathway in B16F10 melanoma cells. Food Chem. Toxicol. 2013, 59, 521-526. [CrossRef]

195. Kim, K.N.; Yang, H.M.; Kang, S.M.; Ahn, G.; Roh, S.W.; Lee, W.; Kim, D.; Jeon, Y.J. Whitening effect of octaphlorethol A isolated from Ishige foliacea in an in vivo zebrafish model. J. Microbiol. Biotechnol. 2015, 25, 448-4451. [CrossRef] [PubMed]

196. del Olmo, A.; Picon, A.; Nuñez, M. High pressure processing for the extension of Laminaria ochroleuca (kombu) shelf-life: A comparative study with seaweed salting and freezing. Innov. Food Sci. Emerg. Technol. 2019, 52, 420-428. [CrossRef]

197. Yang, H.; Liu, D.Q.; Liang, T.J.; Li, J.; Liu, A.H.; Yang, P.; Lin, K.; Yu, X.Q.; Guo, Y.W.; Mao, S.C.; et al. Racemosin C, a novel minor bisindole alkaloid with protein tyrosine phosphatase-1B inhibitory activity from the green alga Caulerpa racemosa. J. Asian Nat. Prod. Res. 2014, 16, 1158-1165. [CrossRef]

198. Ryu, B.; Ahn, B.N.; Kang, K.H.; Kim, Y.S.; Li, Y.X.; Kong, C.S.; Kim, S.K.; Kim, D.G. Dioxinodehydroeckol protects human keratinocyte cells from UVB-induced apoptosis modulated by related genes Bax/Bcl-2 and caspase pathway. J. Photochem. Photobiol. B Biol. 2015, 153, 352-357. [CrossRef]

199. Kang, H.S.; Kim, H.R.; Byun, D.S.; Son, B.W.; Nam, T.J.; Choi, J.S. Tyrosinase inhibitors isolated from the edible brown alga Ecklonia stolonifera. Arch. Pharm. Res. 2004, 27, 1226-1232. [CrossRef] [PubMed]

200. Sappati, P.K.; Nayak, B.; VanWalsum, G.P.; Mulrey, O.T. Combined effects of seasonal variation and drying methods on the physicochemical properties and antioxidant activity of sugar kelp (Saccharina latissima). J. Appl. Phycol. 2019, 31, 1311-1332. [CrossRef]

201. Ko, S.C.; Lee, M.; Lee, J.H.; Lee, S.H.; Lim, Y.; Jeon, Y.J. Dieckol, a phlorotannin isolated from a brown seaweed, Ecklonia cava, inhibits adipogenesis through AMP-activated protein kinase (AMPK) activation in 3T3-L1 preadipocytes. Environ. Toxicol. Pharmacol. 2013, 36, 1253-1260. [CrossRef]

202. Shibata, T.; Fujimoto, K.; Nagayama, K.; Yamaguchi, K.; Nakamura, T. Inhibitory activity of brown algal phlorotannins against hyaluronidase. Eur. J. Phycol. 2002, 37, 493-500. [CrossRef]

203. Aroyehun, A.Q.B.; Razak, S.A.; Palaniveloo, K.; Nagappan, T.; Rahmah, N.S.N.; Jin, G.W.; Chellappan, D.K.; Chellian, J.; Kunnath, A.P. Bioprospecting cultivated tropical green algae, Caulerpa racemosa (Forsskal) J. Agardh: A perspective on nutritional properties, antioxidative capacity and anti-diabetic potential. Foods 2020, 18, 1313. [CrossRef]

204. Saidani, K.; Bedjou, F.; Benabdesselam, F.; Touati, N. Antifungal activity of methanolic extracts of four Algerian marine algae species. Afr. J. Biotechnol. 2012, 11, 9496-9500. [CrossRef] 
205. Manandhar, B.; Paudel, P.; Seong, S.H.; Jung, H.A.; Choi, J.S. Characterizing eckol as a therapeutic aid: A systematic review. Mar. Drugs 2019, 17, 361. [CrossRef] [PubMed]

206. Manandhar, B.; Wagle, A.; Seong, S.H.; Paudel, P.; Kim, H.R.; Jung, H.A.; Choi, J.S. Phlorotannins with potential anti-tyrosinase and antioxidant activity isolated from the marine seaweed Ecklonia stolonifera. Antioxidants 2019, 8, 240. [CrossRef]

207. Kim, S.K. Marine cosmeceuticals. J. Cosmet. Dermatol. 2014, 13, 56-67. [CrossRef]

208. Gheda, S.; Naby, M.A.; Mohamed, T.; Pereira, L.; Khamis, A. Antidiabetic and antioxidant activity of phlorotannins extracted from the brown seaweed Cystoseira compressa in streptozotocin-induced diabetic rats. Environ. Sci. Pollut. Res. Int. 2021, 28, 22886-22901. [CrossRef] [PubMed]

209. Kang, J.I.; Kim, S.C.; Kim, M.K.; Boo, H.J.; Jeon, Y.J.; Koh, Y.S.; Yoo, E.S.; Kang, S.M.; Kang, H.K. Effect of Dieckol, a component of Ecklonia cava, on the promotion of hair growth. Int. J. Mol. Sci. 2012, 13, 6407-6423. [CrossRef] [PubMed]

210. Ferreres, F.; Lopes, G.; Gil-Izquierdo, A.; Andrade, P.B.; Sousa, C.; Mouga, T.; Valentão, P. Phlorotannin extracts from fucales characterized by HPLC-DAD-ESI-MSn: Approaches to hyaluronidase inhibitory capacity and antioxidant properties. Mar. Drugs 2012, 10, 2766-2781. [CrossRef]

211. Sugiura, Y.; Kinoshita, Y.; Misumi, S.; Yamatani, H.; Katsuzaki, H.; Hayashi, Y.; Murase, N. Correlation between the seasonal variations in phlorotannin content and the antiallergic effects of the brown alga Ecklonia cava subs stolonifera. Algal Res. 2021, 58, 102398. [CrossRef]

212. Kim, S.M.; Kang, K.; Jeon, J.S.; Jho, E.H.; Kim, C.Y.; Nho, C.W.; Um, B.H. Isolation of phlorotannins from Eisenia bicyclis and their hepatoprotective effect against oxidative stress induced by tert-butyl hyperoxide. Appl. Biochem. Biotechnol. 2011, 165, 1296-1307. [CrossRef]

213. Catarino, M.D.; Amarante, S.J.; Mateus, N.; Silva, A.; Cardoso, S.M. Brown algae phlorotannins: A marine alternative to break the oxidative stress, inflammation and cancer network. Foods 2021, 10, 1478. [CrossRef]

214. Sugiura, Y.; Takeuchi, Y.; Kakinuma, M.; Amano, H. Inhibitory effects of seaweeds on histamine release from rat basophile leukemia cells (RBL-2H3). Fish Sci. 2006, 72, 1286-1291. [CrossRef]

215. Sugiura, Y.; Matsuda, K.; Yamada, Y.; Nishikawa, M.; Shioya, K.; Katsuzaki, H.; Imai, K.; Amano, H. Isolation of a new anti-allergic phlorotannin, phlorofucofuroeckol-B, from an edible brown alga, Eisenia arborea. Biosci. Biotechnol. Biochem. 2006, 70, $2807-2811$. [CrossRef] [PubMed]

216. Casas, M.P.; Rodríguez-Hermida, V.; Pérez-Larrán, P.; Conde, E.; Liveri, M.T.; Ribeiro, D.; Fernandes, E.; Domínguez, H. In vitro bioactive properties of phlorotannins recovered from hydrothermal treatment of Sargassum muticum. Sep. Purif. Technol. 2016, 167, 117-126. [CrossRef]

217. Eom, S.H.; Lee, E.H.; Park, K.; Kwon, J.Y.; Kim, P.H.; Jung, W.K.; Kim, Y.M. Eckol from Eisenia bicyclis inhibits inflammation through the Akt/NF-kB signaling in Propionibacterium acnes-induced human keratinocyte Hacat cells. J. Food Biochem. 2017, 41, e12312. [CrossRef]

218. Gager, L.; Connan, S.; Molla, M.; Couteau, C.; Arbona, J.F.; Coiffard, L.; Cérantola, S.; Stiger-Pouvreau, V. Active phlorotannins from seven brown seaweeds commercially harvested in Brittany (France) detected by $1 \mathrm{H}$ NMR and in vitro assays: Temporal variation and potential valorization in cosmetic applications. J. Appl. Phycol. 2020, 32, 2375-2386. [CrossRef]

219. Dawczynski, C.; Schubert, R.; Jahreis, G. Amino acids, fatty acids, and dietary fibre in edible seaweed products. Food Chem. 2007, 103, 891-899. [CrossRef]

220. Rhimou, B.; Hassane, R.; José, M.; Nathalie, B. The antibacterial potential of the seaweeds (Rhodophyceae) of the strait of Gibraltar and the mediterranean coast of Morocco. Afr. J. Biotechnol. 2010, 9, 6365-6372.

221. Tapiero, H.; Ba, G.N.; Couvreur, P.; Tew, K.D. Polyunsaturated fatty acids (PUFA) and eicosanoids in human health and pathologies. Biomed. Pharmacother. 2002, 56, 215-222. [CrossRef]

222. Pimentel, F.B.; Alves, R.C.; Rodrigues, F.; Oliveira, M.B.P.P. Macroalgae-derived ingredients for cosmetic industry-An update. Cosmetics 2018, 5, 2. [CrossRef]

223. Kumari, P.; Kumar, M.; Gupta, V.; Reddy, C.R.; Jha, B. Tropical marine macroalgae as potential sources of nutritionally important PUFAs. Food Chem. 2010, 120, 749-757. [CrossRef]

224. Zhou, M.; Yang, M.; Zheng, Y.; Dong, K.; Song, L.; He, C.; Liu, W.; Wang, Y.; Jia, Y. Skin surface lipidomics revealed the correlation between lipidomic profile and grade in adolescent acne. J. Cosmet. Dermatol. 2020, 19, 3349-3356. [CrossRef]

225. Bonnet, C. Lipids, a natural raw material at the heart of cosmetics innovation. OCL 2018, 25, D501. [CrossRef]

226. Bialek, A.; Bialek, M.; Jelinska, M.; Tokarz, A. Fatty acid profile of new promising unconventional plant oils for cosmetic use. Int. J. Cosmet. Sci. 2016, 38, 382-388. [CrossRef] [PubMed]

227. Rabasco Álvarez, A.M.; González Rodríguez, M.L. Lipids in pharmaceutical and cosmetic preparations. Grasas Aceites 2000, 51, 74-96. [CrossRef]

228. Zielińska, A.; Nowak, I. Fatty acids in vegetable oils and their importance in cosmetic industry. CHEMIK nauka-technika-rynek 2014, 68, 103-110.

229. Sanghvi, A.M.; Martin Lo, Y. Present and potential industrial applications of macro-and microalgae. Recent Pat. Food Nutr. Agric. 2010, 2, 187-194. [CrossRef] [PubMed]

230. Wang, R.; Paul, V.J.; Luesch, H. Seaweed extracts and unsaturated fatty acid constituents from the green alga Ulva lactuca as activators of the cytoprotective Nrf2-ARE pathway. Free Radic. Biol. Med. 2013, 57, 141-153. [CrossRef] 
231. Susanto, E.; Fahmi, A.S.; Abe, M.; Hosokawa, M.; Miyashita, K. Lipids, fatty acids, and fucoxanthin content from temperate and tropical brown seaweeds. Aquat. Procedia 2016, 7, 66-75. [CrossRef]

232. Castejón, N.; Thorarinsdottir, K.A.; Einarsdóttir, R.; Kristbergsson, K.; Marteinsdóttir, G. Exploring the potential of icelandic seaweeds extracts produced by aqueous pulsed electric fields-assisted extraction for cosmetic applications. Mar. Drugs 2021, 19, 662. [CrossRef]

233. Plaza, M.; Santoyo, S.; Jaime, L.; Reina, G.G.; Herrero, M.; Señoráns, F.J.; Ibáñez, E. Screening for bioactive compounds from algae. J. Pharm. Biomed. Anal. 2010, 51, 450-455. [CrossRef]

234. Li, T.; Xu, J.; Wu, H.; Jiang, P.; Chen, Z.; Xiang, W. Growth and biochemical composition of Porphyridium purpureum SCS-02 under different nitrogen concentrations. Mar. Drugs 2019, 17, 124. [CrossRef]

235. Neto, R.T.; Marçal, C.; Queirós, A.S.; Abreu, H.; Silva, A.; Cardoso, S.M. Screening of Ulva rigida, Gracilaria s; Fucus vesiculosus and Saccharina latissima as functional ingredients. Int. J. Mol. Sci. 2018, 19, 2987. [CrossRef]

236. Sun, Z.; Mohamed, M.A.; Park, S.Y.; Yi, T.H. Fucosterol protects cobalt chloride induced inflammation by the inhibition of hypoxia-inducible factor through PI3K/Akt pathway. Int. Immunopharmacol. 2015, 29, 642-647. [CrossRef] [PubMed]

237. Hwang, E.; Park, S.Y.; Sun, Z.W.; Shin, H.S.; Lee, D.G.; Yi, T.H. The protective effects of fucosterol against skin damage in UVB-irradiated human dermal fibroblasts. Mar. Biotechnol. 2014, 16, 361-370. [CrossRef] [PubMed]

238. Patra, J.K.; Das, G.; Baek, K.H. Chemical composition and antioxidant and antibacterial activities of an essential oil extracted from an edible seaweed, Laminaria japonica L. Molecules 2015, 20, 12093-12113. [CrossRef] [PubMed]

239. Lee, S.; Lee, Y.S.; Jung, S.H.; Kang, S.S.; Shin, K.H. Anti-oxidant activities of fucosterol from the marine algae Pelvetia siliquosa. Arch. Pharm. Res. 2003, 26, 719-722. [CrossRef] [PubMed]

240. Lee, Y.S.; Jung, S.H.; Lee, S.H.; Shin, K.H. Effects of the extracts from the marine algae Pelvetia siliquosa on hyperlipidemia in rats. Korean J. Pharmacogn. 2004, 35, 143-146.

241. Zhen, X.-H.; Quan, Y.-C.; Jiang, H.-Y.; Wen, Z.-S.; Qu, Y.-L.; Guan, L.-P. Fucosterol, a sterol extracted from Sargassum fusiforme, shows antidepressant and anticonvulsant effects. Eur. J. Pharmacol. 2015, 768, 131-138. [CrossRef]

242. Lee, C.; Park, G.H.; Ahn, E.M.; Kim, B.A.; Park, C.I.; Jang, J.H. Protective effect of Codium fragile against UVB-induced proinflammatory and oxidative damages in HaCaT cells and BALB/c mice. Fitoterapia 2013, 86, 54-63. [CrossRef]

243. Kuda, T.; Ikemori, T. Minerals, polysaccharides and antioxidant properties of aqueous solutions obtained from macroalgal beach-casts in the Noto Peninsula, Ishikawa, Japan. Food Chem. 2009, 112, 575-581. [CrossRef]

244. Rathore, S.S.; Chaudhary, D.R.; Boricha, G.N.; Ghosh, A.; Bhatt, B.P.; Zodape, S.T.; Patolia, J.S. Effect of seaweed extract on the growth, yield and nutrient uptake of soybean (Glycine max) under rainfed conditions. S. Afr. J. Bot. 2009, 75, 351-355. [CrossRef]

245. Mišurcová, L.; Ambrožová, J.; Samek, D. Seaweed lipids as nutraceuticals. Adv. Food Nutr. Res. 2011, 64, 339-355. [CrossRef] [PubMed]

246. Fang, K.S.; Farboud, B.; Nuccitelli, R.; Isseroff, R.R. Migration of human keratinocytes in electric fields requires growth factors and extracellular calcium. J. Invest. Dermatol. 1998, 111, 751-756. [CrossRef]

247. Trevino, M.A.; Herrmann, G.H.; Sprout, W.L. Treatment of severe hydrofluoric acid exposures. J. Occup. Environ. Med. 1983, 25, 861-863. [CrossRef]

248. Muscat, J.E.; Huncharek, M.S. Perineal talc use and ovarian cancer: A critical review. Eur. J. Cancer Prev. 2008, 17, 139-146. [CrossRef] [PubMed]

249. Boisseau, A.M.; Donatien, P.; Surlève-Bazeille, J.E.; Amédée, J.; Harmand, M.F.; Bézian, J.H.; Maleville, J.; Taieb, A. Production of epidermal sheets in a serum free culture system: A further appraisal of the role of extracellular calcium. J. Dermatol. Sci. 1992, 3 , 111-120. [CrossRef]

250. Lansdown, A.B.; Mirastschijski, U.; Stubbs, N.; Scanlon, E.; Ågren, M.S. Zinc in wound healing: Theoretical, experimental, and clinical aspects. Wound Repair Regen 2007, 15, 2-16. [CrossRef]

251. FDA-Food and Drug Administration. Skin protectant drug products for over-the-counter human use; final monograph. Final rule. Fed. Regist. 2003, 68, 33362-33381. Available online: https://www.govinfo.gov/content/pkg/FR-2003-06-04/pdf/03-13751.pdf (accessed on 26 December 2021).

252. Ågren, M.; Chvapil, M.; Franzén, L. Enhancement of re-epithelialization with topical zinc oxide in porcine partial-thickness wounds. J. Surg. Res. 1991, 50, 101-105. [CrossRef]

253. Newman, M.D.; Stotland, M.; Ellis, J.I. The safety of nanosized particles in titanium dioxide-and zinc oxide-based sunscreens. J. Am. Acad. Dermatol. 2009, 61, 685-692. [CrossRef]

254. Bissett, D.L.; McBride, J.F. Iron content of human epidermis from sun-exposed and non-exposed body sites. J. Soc. Cosmet. Chem. 1992, 43, 215-217.

255. Borkow, G.; Gabbay, J.; Lyakhovitsky, A.; Huszar, M. Improvement of facial skin characteristics using copper oxide containing pillowcases: A double-blind, placebo-controlled, parallel, randomized study. Int. J. Cosmet. Sci. 2009, 31, 437-443. [CrossRef] [PubMed]

256. Pence, B.C.; Delver, E.; Dunn, D.M. Effects of dietary selenium on UVB-induced skin carcinogenesis and epidermal antioxidant status. J. Invest. Dermatol. 1994, 102, 759-761. [CrossRef] [PubMed]

257. Burke, K.E. Oral and topical L. selenomethionine protection from ultraviolet-induced sunburn, tanning and skin cancer. $J$. Orthomol. Med. 1992, 7, 83-94. 
258. Burke, K.E.; Burford, R.G.; Combs, G.F., Jr.; French, I.W.; Skeffington, D.R. The effect of topical L-selenomethionine on minimal erythema dose of ultraviolet irradiation in humans. Photodermatol. Photoimmunol. Photomed. 1992, 9, 52-57. [PubMed]

259. McKenzie, R.C.; Beckett, G.J. Mechanisms of selenium-mediated protection from photocarcinogenesis and cell death are not solely p53-dependent. J. Photochem. Photobiol. B Biol. 2003, 71, 99-101. [CrossRef]

260. Sengupta, A.; Lichti, U.F.; Carlson, B.A.; Ryscavage, A.O.; Gladyshev, V.N.; Yuspa, S.H.; Hatfield, D. Selenoproteins Are Essential for Proper Keratinocyte Function and Skin Development. PLoS ONE 2010, 5, e12249. [CrossRef]

261. Klaschka, U. Natural personal care products-Analysis of ingredient lists and legal situation. Environ. Sci. Eur. 2016, 28, 8. [CrossRef]

262. Marinoa, T.; Iovinea, A.; Casellab, P.; Martinoc, M.; Chianesea, S.; Laroccac, V.; Musmarra, D.; Molino, A. From Haematococcus pluvialis microalgae a powerful antioxidant for cosmetic applications. Chem. Eng. 2020, 79, 271-276.

263. Thiyagarasaiyar, K.; Goh, B.H.; Jeon, Y.J.; Yow, Y.Y. Algae metabolites in cosmeceutical: An overview of current applications and challenges. Mar. Drugs 2020, 18, 323. [CrossRef]

264. Gade, R.; Tulasi, M.S.; Bhai, V.A. Seaweeds: A novel biomaterial. Int. J. Pharm. Pharm. Sci. 2013, 5, 975-1491.

265. Álvarez-Gómez, F.; Korbee, N.; Casas-Arrojo, V.; Abdala-Díaz, R.T.; Figueroa, F.L. UV photoprotection, cytotoxicity and immunology capacity of red algae extracts. Molecules 2019, 24, 341. [CrossRef] [PubMed]

266. Fabrowska, J.; Leska, B.; Schroeder, G.; Messyasz, B.; Pikosz, M. Biomass and extracts of algae as materials for cosmetics. In Marine Algae Extracts, Processes, Products and Applications; Kim, S., Chojnacka, K., Eds.; Wiley-VCH Verlag GmbH \& Co.: Weinheim, Germany, 2015; pp. 681-706.

267. Olasehinde, T.A.; Olaniran, A.O.; Okoh, A.I. Therapeutic potentials of microalgae in the treatment of Alzheimer's disease. Molecules 2017, 22, 480. [CrossRef] [PubMed]

268. Ribeiro, A.; Estanqueiro, M.; Oliveira, M.; Sousa Lobo, J. Main benefits and applicability of plant extracts in skin care products. Cosmetics 2015, 2, 48-65. [CrossRef]

269. Khan, M.I.; Shin, J.H.; Kim, J.D. The promising future of microalgae: Current status, challenges, and optimization of a sustainable and renewable industry for biofuels, feed, and other products. Microb. Cell Factories 2018, 17, 36. [CrossRef]

270. Sebök, S.; Herppich, W.B.; Hanelt, D. Outdoor cultivation of Ulva lactuca in a recently developed ring-shaped photobioreactor: E_ects of elevated $\mathrm{CO}_{2}$ concentration on growth and photosynthetic performance. Botanica Mar. 2019, 62, 179-190. [CrossRef]

271. Kadam, S.U.; Tiwari, B.K.; O’Donnell, C.P. Application of novel extraction technologies for bioactives from marine algae. J. Agric. Food Chem. 2013, 61, 4667-4675. [CrossRef] [PubMed]

272. Lou, H.Y.; Wang, B.; Yu, C.G.; Xu, Y.F. Optimization of microwave-assisted extraction of polyphenols from Enteromorpha prolifera by orthogonal test. Chin. Herb. Med. 2010, 2, 321-325.

273. Rodriguez-Jasso, R.M.; Mussatto, S.I.; Pastrana, L.; Aguilar, C.N.; Teixeira, J.A. Microwave-assisted extraction of sulfated polysaccharides (fucoidan) from brown seaweed. Carbohydr. Polym. 2011, 86, 1137-1144. [CrossRef]

274. Cikoš, A.M.; Jokic, S.; Šubaric, D.; Jerkovic, I. Overview on the application of modern methods for the extraction of bioactive compounds from marine macroalgae. Mar. Drugs 2018, 16, 348. [CrossRef]

275. Sivagnanam, S.P.; Yin, S.; Choi, J.H.; Park, Y.B.; Woo, H.C.; Chun, B.S. Biological properties of fucoxanthin in oil recovered from two brown seaweeds using supercritical $\mathrm{CO}_{2}$ extraction. Mar. Drugs 2015, 13, 3422-3442. [CrossRef]

276. Mittal, R.; Tavanandi, H.A.; Mantri, V.A.; Raghavarao, K.S.M.S. Ultrasound assisted methods for enhanced extraction of Phycobiliproteins from marine macro-algae, Gelidium pusillum (Rhodophyta). Ultrason. Sonochem. 2017, 38, 92-103. [CrossRef] [PubMed]

277. Collett, M.G. Photosensitisation diseases of animals: Classification and a weight of evidence approach to primary causes. Toxicon X 2019, 3, 100012. [CrossRef] [PubMed]

278. Anderlova, D.; Pšurný, M. Exploring the importance of emotions within consumer behaviour on the Czech luxury cosmetic market. Acta Univ. Agric. Silvicult. Mendel. Brun. 2020, 68, 363-372. [CrossRef] 